\title{
Entropy Measures for Atanassov intuitionistic fuzzy sets based on divergence
}

\author{
Ignacio Montes · Nikhil R. Pal · Susana Montes
}

Received: date / Accepted: date

\begin{abstract}
In the literature, there are two different approaches to define entropy of Atanassov Intuitionistic Fuzzy Sets (AIFS, for short). The first approach, given by Szmidt and Kacprzyk, measures how far is an AIFS from its closest crisp set, while the second approach, given by Burrillo and Bustince, measures how far is an AIFS from its closest fuzzy set. On the other hand, divergence measures are functions that measure how different two AIFSs are. This paper studies how to define both types of entropies using measures of local divergence. In this context we provide the necessary and sufficient conditions for defining entropy measures under both frameworks using divergence measures for AIFS. We also show that the usual examples of entropy measures can be obtained as a particular case of our more general framework. Also, we investigate the connection between knowledge measures and divergence measures. Finally, we apply our results in a multi-attribute decision making problem to obtain the weights of the experts.
\end{abstract}

Keywords Atanassov Intuitionistic Fuzzy Sets . Divergence measures · Entropy

\section{Montes}

Dep. of Statistics and O.R., University of Oviedo, Spain

Tel.: +34 985103357

E-mail: imontes@uniovi.es

N.R. Pal

Electronics and Communication Sciences Unit, Indian Statistical Institute, India

E-mail: nrpal159@gmail.com

S. Montes

Dep. of Statistics and O.R., University of Oviedo, Spain

Tel.: +34985102133

E-mail: montes@uniovi.es

\section{Introduction}

Fuzzy sets were introduced by Zadeh in 1965 [?] to model situations where the available information is vague or incomplete. For any element, a fuzzy set is characterized by a membership degree, which indicates the degree to which the element belongs to the set or satisfies the property described by the fuzzy set. The theory of fuzzy sets has been widely studied both from the theoretical and applied points of view (see [?,?,?], among others).

Over the years, several extensions of fuzzy sets have been proposed: interval valued fuzzy sets, type-2 fuzzy sets [?], hesitant fuzzy sets [?], and so on. In 1986, Atanassov [?] proposed the notion of Intuitionistic Fuzzy Set (AIFS, for short). The idea is quite simple: for any element, an AIFS assigns a membership and a nonmembership degree. The former represents the degree to which an element belongs to the set or complies with the property described by the set, while the latter represents the degree to which the element does not belong to the set. The membership and non-membership degrees satisfy a mathematical constraint: their sum cannot exceed one. The difference between one and the sum of both degrees is called hesitation index, which represents the lack of knowledge about whether the element belongs or not to the set. In recent past, research work on the theory of Atanassov IFSs has exponentially grown, and it has been successfully applied in decision making [?,?,?], pattern recognition [?,?] and image segmentation [?], among others.

For developing useful applications, two important lines of research have attracted the attention of the researchers. One of the approaches involves comparison of AIFSs. In this framework, many different measures of comparison have been suggested in the liter- 
ature, like distances or dissimilarities. However, it can be argued that these measures could be inadequate in some context. For this reason, we have introduced divergence measures in our previous works [?,?], and we have shown many interesting mathematical properties as well as their usefulness in many applications [?,?]. The other approach involves the study of entropies for AIFSs. In this framework, two different trends can be found: (i) The Szmidt and Kacprzyk [?] approach, which considers the entropy as a measure of fuzziness: it measures how distant is an AIFS to be a crisp set. (ii) The Burrillo and Bustince [?] approach, which interprets entropies as measures of intuitionism: it measures how different is an AIFS to be a fuzzy set.

Our aim in this paper is to define entropies, both Szmidt and Kacprzyk (SK) type and Burrillo and Bustince (BB) type, using divergences. For this, after introducing some preliminary notations in Section 2, in Sections 3 and 4 we study how measures of divergence can be used to define entropies under both frameworks. In Section 5 we investigate the connection between divergences and knowledge measures [?]. We provide some concluding remarks in Section 7. Preliminary results of this investifation has been included in [?].

\section{Preliminaries}

In this section we introduce the main notions used in this paper. First of all, we introduce fuzzy sets and AIFSs. We also explain a graphical interpretation of AIFSs and define some usual operations between these sets. Then, we recall the definition of divergences for fuzzy sets [?] and AIFSs [?], emphasizing the property of locality [?]. We conclude this section explaining in detail the primary objective of this paper.

Throughout this paper, we consider a finite universe $X$ whose cardinality will be denoted by $n$, that is, $|X|=$ $n$.

\subsection{Atanassov Intuitionistic Fuzzy Sets}

Fuzzy sets were introduced by Zadeh [?] as an extension of crisp sets to model vague or linguistic information. While a crisp set $A$ only allows two possibilities $x \in$ $A$ or $x \notin A$, a fuzzy set $A$ allows, for any $x \in X$, a degree of membership of the element $x$ to the set. This membership degree, formally defined as a function $\mu_{A}$ : $X \rightarrow[0,1]$, represents the degree in which an element belongs to $A$, or the degree to which it satisfies the property described by the set $A$. In this way, $1-\mu_{A}(x)$ represents the degree to which $x$ does not belong to $A$.
In [?], Atanassov discussed that the non-membership degree could be different than $1-\mu_{A}(x)$ due to lack of knowledge. To account for this, he proposed an extension of fuzzy sets allowing two degrees: the membership and non-membership degrees, which correspond to the degree to which an element belongs and does not belong to the set, respectively. Formally, an intuitionistic fuzzy set, AIFS for short, is defined by $A=$ $\left\langle\left(x, \mu_{A}(x), \nu_{A}(x)\right) \mid x \in X\right\rangle$, where $\mu_{A}(x)$ and $\nu_{A}(x)$ denote the membership and non-membership degrees of $x$ to $A$, respectively. Any AIFS has an associated hesitation index, denoted by $\pi_{A}$, and defined by $\pi_{A}(x)=$ $1-\mu_{A}(x)-\nu_{A}(x)$ for any $x \in X$, and it measures the lack of knowledge about whether $x$ belongs or not to $A$.

Any fuzzy set $A$ can be expressed as an AIFS, just by taking $\nu_{A}=1-\mu_{A}$. Therefore, for fuzzy sets it holds that $\pi_{A}=0$. Also, a crisp set is a particular case of an AIFS where for any $x \in X$, either $\mu_{A}(x)=1$ and $\nu_{A}(x)=0$, if $x \in A$, or $\mu_{A}(x)=0$ and $\nu_{A}(x)=1$, if $x \notin A$. From now on, we denote by $\operatorname{AIFS}(X)$ the set of all AIFSs on $X$, and by $\mathrm{FS}(X)$ the set of all fuzzy sets on $X$.

Any AIFS can be graphically depicted for any $x \in X$ as a pair $\left(\mu_{A}(x), \nu_{A}(x)\right)$, as can be seen in Figure 1 . The segment that goes from $(1,0)$ to $(0,1)$ corresponds to the pairs $\left(\mu_{A}(x), \nu_{A}(x)\right)$ where $\nu_{A}(x)=1-\mu_{A}(x)$. If for any $x \in X$, the element $\left(\mu_{A}(x), \nu_{A}(x)\right)$ belongs to such segment, $A$ is a fuzzy set. Moreover, the further $\left(\mu_{A}(x), \nu_{A}(x)\right)$ is from the segment $((1,0),(0,1))$, the greater is the hesitation index $\pi_{A}(x)$. Also, the case of total ignorance, that is when $\pi_{A}(x)=1$, corresponds to the pair $(0,0)$.

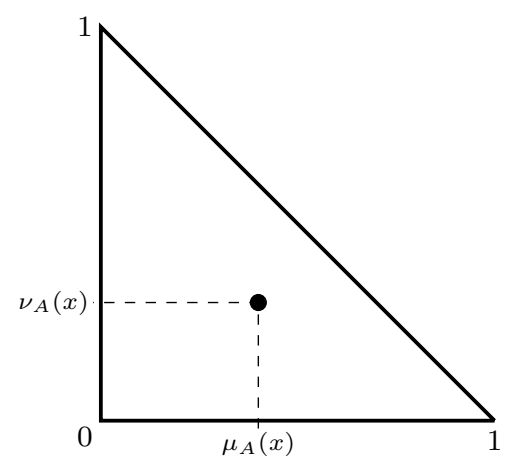

Fig. 1: Graphical representation of AIFSs.

To conclude this subsection, let us recall some basic operations between AIFSs. Given $A, B \in \operatorname{AIFS}(X)$, we consider the following operations:

- The union of $A$ and $B$, denoted by $A \cup B$, is the AIFS whose membership and non-membership degrees are 
given by:

$$
\begin{aligned}
\mu_{A \cup B}(x) & =\max \left\{\mu_{A}(x), \mu_{B}(x)\right\}, \\
\nu_{A \cup B}(x) & =\min \left\{\mu_{A}(x), \mu_{B}(x)\right\} .
\end{aligned}
$$

- The intersection of $A$ and $B$, denoted by $A \cap B$, is the AIFS whose membership and non-membership degrees are given by:

$$
\begin{aligned}
& \mu_{A \cap B}(x)=\min \left\{\mu_{A}(x), \mu_{B}(x)\right\}, \\
& \nu_{A \cap B}(x)=\max \left\{\mu_{A}(x), \mu_{B}(x)\right\} .
\end{aligned}
$$

- $A$ is included in $B$, denoted by $A \subseteq B$, if $\mu_{A} \leq \mu_{B}$ and $\nu_{A} \geq \nu_{B}$.

- The complement of $A$, denoted by $A^{c}$, is defined by:

$$
A^{c}=\left\langle\left(x, \nu_{A}(x), \mu_{A}(x)\right) \mid x \in X\right\rangle .
$$

We note here that more general families of unions and intersections can be defined using a T-norm for min and T-conrom (S-norm) for max.

\subsection{Divergences for AIFSs}

One very popular topic of research within AIFSs theory is that of measuring how different two AIFSs are. Although there are many different approaches, for instance similarities or distances, we have introduced in [?] a new family of measures, for comparing this kind of sets, called AIF-divergences, and we have argued that, from our point of view, AIF-divergences are more apropriate than other measures of comparison existing in the literature.

Definition 1 ([?]) A function $D$ defined from $\operatorname{AIFS}(X) \times$ $\operatorname{AIFS}(X)$ to $\mathbb{R}^{+}$is an AIF-divergence if it satisfies the following properties:

(Div.1) $D(A, B)=D(B, A)$ for any $A, B \in \operatorname{AIFS}(X)$.

(Div.2) $D(A, A)=0$ for any $A \in \operatorname{AIFS}(X)$.

(Div.3) $D(A \cap C, B \cap C) \leq D(A, B)$ for any $A, B, C \in$ $\operatorname{AIFS}(X)$.

(Div.4) $D(A \cup C, B \cup C) \leq D(A, B)$ for any $A, B, C \in$ $\operatorname{AIFS}(X)$.

Hence, an AIF-divergence is symmetric, takes the value 0 when comparing an AIFS with itself, and the closer two AIFSs are, the smaller the AIF-divergence between them.

In [?, Lemma 3.2] we proved that any AIF-divergence satisfies the following property, which will be useful for the forthcoming results:

$$
A \subseteq B \subseteq C \Rightarrow D(A, C) \geq \max \{D(A, B), D(B, C)\}
$$

One particular family of AIF-divergences that satisfies the local property is:

$$
\begin{aligned}
D(A \cup\{x\}, B \cup\{x\})-D(A, B) & \\
& =h_{\mathrm{IF}}\left(\mu_{A}(x), \nu_{A}(x), \mu_{B}(x), \nu_{B}(x)\right) ;
\end{aligned}
$$

where $h_{\mathrm{IF}}$ satisfies five locality properties in Theorem 1. This means that if we modify the membership and nonmembership degrees of only one element of the sets, the change in the AIF-divergence between the sets depends only on what has been changed.

In [?] we characterized local AIF-divergences, using the following notation ${ }^{1}$ :

$\mathcal{D}=\left\{\left(u_{1}, u_{2}, v_{1}, v_{2}\right) \in \mathbb{R}^{4^{+}} \mid u_{1}+u_{2} \leq 1, v_{1}+v_{2} \leq 1\right\}$.

Theorem 1 [?] An AIF-divergence $D$ is local if and only if there exists a function $h: \mathcal{D} \rightarrow \mathbb{R}^{+}$such that

$D(A, B)=\sum_{x \in X} h_{\mathrm{IF}}\left(\mu_{A}(x), \nu_{A}(x), \mu_{B}(x), \nu_{B}(x)\right)$

and satisfying the following properties:

(AIF-loc. 1) $h_{\mathrm{IF}}(u, v, u, v)=0$ for any $(u, v, u, v) \in \mathcal{D}$.

(AIF-loc.2) $h_{\mathrm{IF}}\left(u_{1}, u_{2}, v_{1}, v_{2}\right)=h_{\mathrm{IF}}\left(v_{1}, v_{2}, u_{1}, u_{2}\right)$ for any $\left(u_{1}, u_{2}, v_{1}, v_{2}\right) \in \mathcal{D}$.

(AIF-loc.3) If $\left(u_{1}, u_{2}, v_{1}, v_{2}\right) \in \mathcal{D}, \omega \in[0,1]$ and $u_{1} \leq$ $\omega \leq v_{1}$, it holds that:

$h_{\mathrm{IF}}\left(u_{1}, u_{2}, v_{1}, v_{2}\right) \geq h_{\mathrm{IF}}\left(u_{1}, u_{2}, \omega, v_{2}\right)$.

Moreover, if $\max \left\{u_{2}, v_{2}\right\}+\omega \leq 1$, it holds that:

$h_{\mathrm{IF}}\left(u_{1}, u_{2}, v_{1}, v_{2}\right) \geq h_{\mathrm{IF}}\left(\omega, u_{2}, v_{1}, v_{2}\right)$.

(AIF-loc.4) If $\left(u_{1}, u_{2}, v_{1}, v_{2}\right) \in \mathcal{D}, \omega \in[0,1]$ and $u_{2} \leq$ $\omega \leq v_{2}$, it holds that:

$h_{\mathrm{IF}}\left(u_{1}, u_{2}, v_{1}, v_{2}\right) \geq h_{\mathrm{IF}}\left(u_{1}, u_{2}, v_{1}, \omega\right)$.

Moreover, if $\max \left\{u_{1}, v_{1}\right\}+\omega \leq 1$, it holds that:

$h_{\mathrm{IF}}\left(u_{1}, u_{2}, v_{1}, v_{2}\right) \geq h_{\mathrm{IF}}\left(u_{1}, \omega, v_{1}, v_{2}\right)$.

(AIF-loc.5) If $\left(u_{1}, u_{2}, v_{1}, v_{2}\right) \in \mathcal{D}$ and $\omega \in[0,1]$, then if $\max \left\{u_{2}, v_{2}\right\}+\omega \leq 1$ it holds that:

$h_{\mathrm{IF}}\left(\omega, u_{2}, \omega, v_{2}\right) \leq h_{\mathrm{IF}}\left(u_{1}, u_{2}, v_{1}, v_{2}\right)$

and if $\max \left\{u_{1}, v_{1}\right\}+\omega \leq 1$, it holds that:

$h_{\mathrm{IF}}\left(u_{1}, \omega, v_{1}, \omega\right) \leq h_{\mathrm{IF}}\left(u_{1}, u_{2}, v_{1}, v_{2}\right)$.

1 The notation we are using here is slightly different from that on [?], where the set $\mathcal{D}$ was defined by $\mathcal{D}=\{(u, v) \in$ $\left.\mathbb{R}^{2+} \mid u+v \leq 1\right\}$, and then we considered $\mathcal{D}^{2}$. In this paper, we have considered the alternative expression for $\mathcal{D}$ in Equation (2) for the sake of mathematical convenience. However, both approaches are equivalent. 
Let us prove a useful property of the function $h_{\mathrm{IF}}$ associated with a local AIF-divergence.

Proposition 1 Let $D$ be a local AIF-divergence with associated function $h_{\mathrm{IF}}$. Then, $h_{\mathrm{IF}}(u, v, 1,0)$ is decreasing on $u$ and increasing on $v$, and $h_{\mathrm{IF}}(u, v, 0,1)$ is increasing on $u$ and decreasing on $v$, whenever $u+v \leq 1$.

Proof Let us prove that $h_{\mathrm{IF}}(u, v, 1,0)$ is decreasing in $u$. For this, take $u_{1} \leq u_{2}$ such that $u_{2}+v \leq 1$. Taking property (AIF-loc.3) into account, it holds that:

$h_{\mathrm{IF}}\left(u_{1}, v, 1,0\right) \geq h_{\mathrm{IF}}\left(u_{2}, v, 1,0\right)$.

On the other hand, let us see that $h_{\mathrm{IF}}(u, v, 1,0)$ is increasing in $v$. For this, take $v_{1} \leq v_{2}$. Define the AIFSs $A, B, M$ on $\{x\}$ by:

$A=\left\langle\left(x, u, v_{1}\right)\right\rangle, \quad B=\left\langle\left(x, u, v_{2}\right)\right\rangle, \quad M=\langle(x, 1,0)\rangle$.

From property (Div.4), $D(A, M)=D(A \cup B, A \cup M) \leq$ $D(B, M)$, which means that:

$h_{\mathrm{IF}}\left(u, v_{2}, 1,0\right) \geq h_{\mathrm{IF}}\left(u, v_{1}, 1,0\right)$.

Let us now study the function $h_{\mathrm{IF}}(u, v, 0,1)$. First of all, let us see that it is increasing in the first component. Take $u_{1} \leq u_{2}$, and define the AIFSs $A, B, N$ on $\{x\}$ by: $A=\left\langle\left(x, u_{2}, v\right)\right\rangle, \quad B=\left\langle\left(x, u_{1}, v\right)\right\rangle, \quad N=\langle(x, 0,1)\rangle$.

Using property (Div.3), we obtain that

$D(B, N)=D(A \cap B, N \cap B) \leq D(A, N)$,

which means that:

$h_{\mathrm{IF}}\left(u_{2}, v, 0,1\right) \geq h_{\mathrm{IF}}\left(u_{1}, v, 0,1\right)$.

On the other hand, let us see that $h_{\mathrm{IF}}(u, v, 0,1)$ is decreasing in $v$. Taking $v_{1} \leq v_{2}$ and using (AIF-loc.4) it holds that:

$h_{\mathrm{IF}}\left(u, v_{1}, 0,1\right) \geq h_{\mathrm{IF}}\left(u, v_{2}, 0,1\right) . \square$

Divergences for fuzzy sets had already been introduced in [?]. A function $D: \operatorname{FS}(X) \times \operatorname{FS}(X) \rightarrow \mathbb{R}$ is a $d i$ vergence for fuzzy sets if it satisfies conditions (Div.1) to (Div.4) when we restrict them to $\mathrm{FS}(X)$. The property of locality has also been defined for divergences for fuzzy sets, and it was characterized in the following way:

Theorem 2 ([?, Prop. 3.4]) A function $D: \operatorname{FS}(X) \times$ $\mathrm{FS}(X) \rightarrow \mathbb{R}^{+}$is a local divergence for fuzzy sets if and only if there exists a function $h_{\mathrm{FS}}:[0,1] \times[0,1] \rightarrow \mathbb{R}^{+}$ such that:

$D(A, B)=\sum_{x \in \mathcal{X}} h_{\mathrm{FS}}\left(\mu_{A}(x), \mu_{B}(x)\right)$

and it satisfies the following properties:

(loc.1) $h_{\mathrm{FS}}(u, v)=h_{\mathrm{FS}}(v, u)$ for any $(u, v) \in[0,1]^{2}$.

(loc.2) $h_{\mathrm{FS}}(u, u)=0$ for any $u \in[0,1]$.

(loc.3) $h_{\mathrm{FS}}(u, z) \geq \max \left\{h_{\mathrm{FS}}(u, v), h(v, z)\right\}$ for any $u$, $v, z \in[0,1]$ such that $u<v<z$.

\subsection{Divergence-based Entropies of AIFS}

In the fuzzy framework, the notion of entropy or fuzziness was introduced by De Luca and Termini [?]. Since then, many researchers continued working on this topic, such as [?,?,?,?,?,?], among others. In particular, Montes et al. [?] used local divergences for fuzzy sets as a measure of entropy or fuzziness.

Our objective here is to define entropies for AIFSs by using local AIF-divergences. As we shall explain later, there are two different types of entropies: the one defined by Szmidt and Kacprzyk [?], that measures how different is an AIFS from its nearest crisp set, and the one defined by Burrillo and Bustince [?] that measures how different is an AIFS from its closest fuzzy set. As Pal et al. have already explained in [?], both types of entropies are different and can be interpreted as complementary.

From now on, we consider a local AIF-divergence $D$ with associated function $h_{\mathrm{IF}}$, and we investigate the additional properties that must be imposed on $h_{\mathrm{IF}}$ to define entropies, with respect to both interpretations, the interpretation of Szmidt and Kacprzyk (SK) and that of Burrillo and Bustince (BB).

For this we make two assumptions: (i) the local AIFdivergence can be expressed by:

$D(A, B)=\frac{1}{n} \sum_{x \in X} h_{\mathrm{IF}}\left(\mu_{A}(x), \nu_{A}(x), \mu_{B}(x), \nu_{B}(x)\right)$

for any $A, B \subseteq A I F S(X)$; and (ii) $h_{\mathrm{IF}}$ takes values in $[0,1]$, meaning that for any element the maximal difference is 1 . As far as $D$ is upper bounded, the previous assumptions can be simply understood as a rescaling of the divergence. Therefore, these assumptions are only made for mathematical convenience.

\section{SK-Entropies}

Here we deal with entropies measuring how far is an AIFS to be a crisp set, which will be called SK-entropies. Following the definition of entropy given by Szmidt and Kacprzyk [?], we introduce the notion of closest crisp to an AIFS, and then use local AIF-divergences to define SK-entropies.

\subsection{Szmidt and Kacprzyk's Entropy}

We start introducing the definition of entropy for AIFSs given by Szmidt and Kacprzyk [?]. The idea behind this definition is quite simple: it measures how far is an AIFS from its closest crisp set. 
Definition 2 ([?]) A function $E: \operatorname{AIFS}(X) \rightarrow[0,1]$ is an entropy if it satisfies the following axioms:

( $\left.I_{\mathrm{SK}} 1\right) E(A)=0$ if and only if $A$ is a crisp set.

$\left(I_{\mathrm{SK}} 2\right) E(A)=1$ if and only if $\mu_{A}(x)=\nu_{A}(x)$ for every $x \in X$.

$\left(I_{\mathrm{SK}} 3\right) E(A)=E\left(A^{c}\right)$.

$\left(I_{\mathrm{SK}} 4\right) E(A) \leq E(B)$ if $\mu_{A}(x) \leq \mu_{B}(x)<\nu_{B}(x) \leq$ $\nu_{A}(x)$ or $\nu_{A}(x) \leq \nu_{B}(x) \leq \mu_{B}(x) \leq \mu_{A}(x)$ for every $x \in X$.

Let us discuss the previous conditions. The condition ( $\left.I_{\mathrm{SK}} 1\right)$ implies that the entropy is zero (non-existent) if, and only if, the set is crisp. $\left(I_{\mathrm{SK}} 3\right)$ says that the entropy is closed with respect to the complement. According to, Property $\left(I_{\mathrm{SK}} 2\right)$, the entropy takes the maximum value if, and only if, both the membership and non-membership degrees coincide. However, in some applications this property can be argued to be rather soft: given two AIFSs $A$ and $B$ satisfying $\mu_{A}=\nu_{A}$ and $\mu_{B}=\nu_{B}$, both sets have the same entropy, regardless of exact values of the membership degrees $\mu_{A}$ and $\mu_{B}$ (or $\pi_{A}$ and $\left.\pi_{B}\right)$. Hence, property $\left(I_{\mathrm{SK}} 2\right)$ does not take into account the hesitation index associated with the AIFSs. For example, consider the AIFSs $A$ and $B$ defined by:

$A=\langle(x, 0.1,0.1) \mid \forall x \in X\rangle$,

$B=\langle(x, 0.45,0.45) \mid \forall x \in X\rangle$.

From $\left(I_{\mathrm{SK}} 2\right), E$ satisfies $E(A)=E(B)$, but the lack of information associated with $A$ seems to be greater than that with $B$, because $\pi_{A}(x)=0.8>0.1=\pi_{B}(x)$ for any $x \in X$. These AIFSs have been graphically depicted in Figure 2.

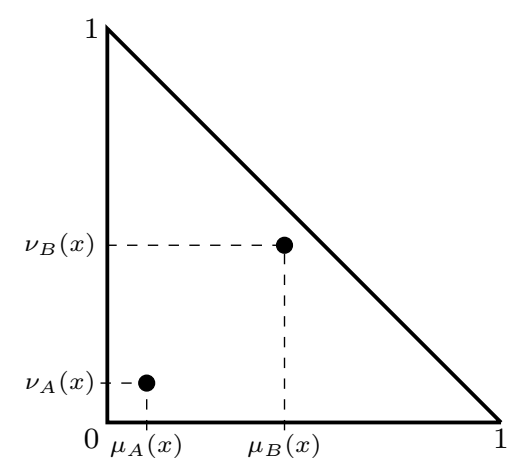

Fig. 2: Graphical representation of the AIFSs in Equation (3).

Property $\left(I_{\mathrm{SK}} 2\right)$ can be slightly modified in order to avoid this drawback as follows:
$\left(I_{\mathrm{SK}} 2^{\prime}\right) E(A)=1$ if and only if $\mu_{A}(x)=\nu_{A}(x)=0$ for every $x \in X$.

This is more plausible as it implies that $E(A)=1$ when we have no knowledge about membership and non-membership for every element. This modified property can be equivalently expressed in terms of the hesitation index, since $E(A)=1$ if and only if $\pi_{A}=1$. For the AIFSs defined in Equation (3), if we consider property $\left(I_{\mathrm{SK}} 2^{\prime}\right)$, an entropy must satisfy $E(A) \geq E(B)$, but the equality is not required.

Finally, condition $\left(I_{\mathrm{SK}} 4\right)$ says that the closer is the set to be a crisp set, the lower is its entropy.

Taking into account Definition 2 as well as the previous discussion, we consider the following definition of a SK-entropy.

Definition 3 A mapping $E: \operatorname{AIFS}(X) \rightarrow[0,1]$ is a $S K$-entropy if it satisfies the properties $\left(I_{\mathrm{SK}} 1\right),\left(I_{\mathrm{SK}} 2^{\prime}\right)$, $\left(I_{\mathrm{SK}} 3\right)$ and $\left(I_{\mathrm{SK}} 4\right)$.

\subsection{SK-entropies based on local AIF-divergences}

Throughout this subsection we aim to investigate how SK-entropies can be built using local AIF-divergences. For this aim, we first introduce the notion of closest crisp set to an AIFS.

Definition 4 Given $A \in \operatorname{AIFS}(X)$, we define the closest crisp set to $A$, denoted by $C_{A}$, by:

$x \in C_{A}$ if $\mu_{A}(x) \geq \nu_{A}(x)$, and $x \notin C_{A}$ otherwise.

This notion had already been considered for fuzzy sets in [?].

Since any crisp set is an AIFS with zero hesitation index, we can express the closet crisp set to $A$ as:

$\mu_{C_{A}}(x)= \begin{cases}1 & \text { if } \mu_{A}(x) \geq \nu_{A}(x), \\ 0 & \text { otherwise }\end{cases}$

and

$\nu_{C_{A}}(x)= \begin{cases}0 & \text { if } \mu_{A}(x) \geq \nu_{A}(x), \\ 1 & \text { otherwise }\end{cases}$

In Figure 3 we can depict an example of the closest crisp set to an AIFS. In the left panel of the picture, we show an example where $\mu_{A}(x)<\nu_{A}(x)$, so $x \notin C_{A}$, or equivalently, $\mu_{C_{A}}(x)=0, \nu_{C_{A}}(x)=1$. The opposite happens in the right panel of the picture, where $\mu_{B}(x)>\nu_{B}(x)$, so $x \in C_{B}$, or equivalently, $\mu_{C_{B}}(x)=1, \nu_{C_{B}}(x)=0$. As we can see in the picture, as long as $\left(\mu_{A}(x), \nu_{A}(x)\right)$ is above the dotted line which represents the pairs $(t, t)$, $x \notin C_{A}$, while as long as $\left(\mu_{B}(x), \nu_{B}(x)\right)$ is on or below the dotted line, $x \in C_{B}$.

Next proposition shows two simple but useful properties of the closest crisp set to an AIFS. 


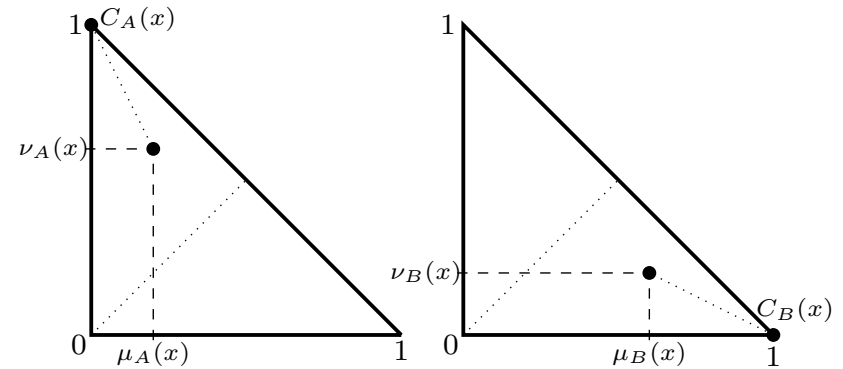

Fig. 3: Example of the closest crisp set to an AIFS.

Proposition 2 Consider $A \in \operatorname{AIFS}(X)$, and let $C_{A}$ be its closest crisp set. The following statements hold:

1. A is a crisp set if and only if $A=C_{A}$.

2. For any $x \in X$, if $\mu_{A}(x) \neq \nu_{A}(x)$, then $C_{A}^{c}(x)=$ $C_{A^{c}}(x)$, where $C_{A^{c}}$ denotes the closets crisp set to $A^{c}$.

Proof Let us prove the first item. Obviously, if $A=C_{A}$, $A$ is a crisp set. On the other hand, if $A$ is a crisp set, for any $x \in X$ either $x \in A$, which implies $\mu_{A}(x)=1$, or $x \notin A$, which implies $\nu_{A}(x)=1$. In the former case, $\mu_{A}(x)>\nu_{A}(x)$, and therefore $x \in C_{A}$, while in the second case $\mu_{A}(x)<\nu_{A}(x)$, which implies $x \notin C_{A}$. We conclude that $A=C_{A}$.

Let us now turn to the second item. Since $\mu_{A}(x) \neq$ $\nu_{A}(x)$, we only have two possibilities, either $\mu_{A}(x)>$ $\nu_{A}(x)$ or $\mu_{A}(x)<\nu_{A}(x)$. Assume we are in the former case. By definition of $C_{A}$, it holds that $\mu_{C_{A}}(x)=$ $1, \nu_{C_{A}}(x)=0$, which implies that $x \in C_{A}$, and consequently $x \notin C_{A}^{c}$. Also, since $\mu_{A}(x)>\nu_{A}(x)$, it holds that:

$\mu_{A^{c}}(x)=\nu_{A}(x)<\mu_{A}(x)=\nu_{A^{c}}(x)$,

so $x \notin C_{A^{c}}$.

On the other hand, if $\nu_{A}(x)>\mu_{A}(x)$, following a similar reasoning we obtain that $x \in C_{A}^{c}$ and $x \in C_{A^{c}}$, so we conclude that $C_{A}^{c}(x)=C_{A^{c}}(x)$.

From the second item, we deduce that when $\mu_{A}(x) \neq$ $\nu_{A}(x), \mu_{C_{A^{c}}}(x)=\nu_{C_{A}}(x)$ and $\nu_{C_{A^{c}}}(x)=\mu_{C_{A}}(x)$.

The second item of Proposition 2 is graphically explained in Figure 4. As it can be seen in the left-side panel, when $\mu_{A}(x)>\nu_{A}(x), C_{A}(x)$ and $C_{A^{c}}(x)$ are just the opposite, so $C_{A}^{c}(x)=C_{A^{c}}(x)$. On the other hand, note that the second item requires $\mu_{A}(x) \neq \nu_{A}(x)$. The reason is that if $\mu_{A}(x)=\nu_{A}(x)$ holds, as in the rightside panel, the closest crisp set to $A$ and $A^{c}$ in $x$ coincide as is equal to $\mu_{C_{A}}(x)=1, \nu_{C_{A}}(x)=0$. That is why condition $\mu_{A}(x) \neq \nu_{A}(x)$ is required in the second item of the Proposition 2.

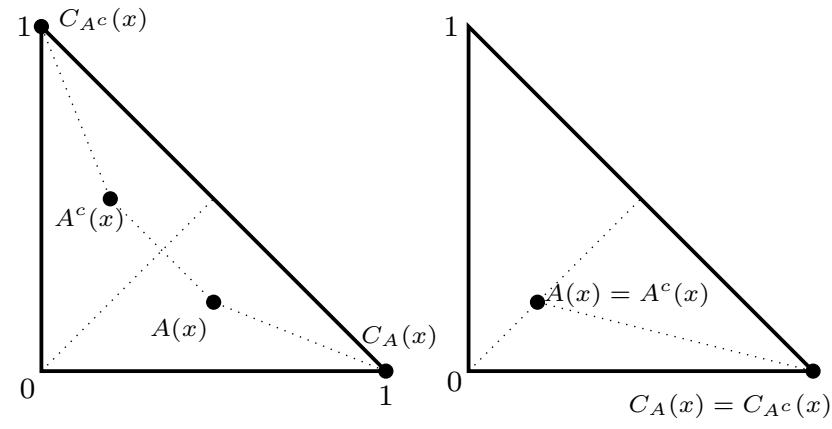

Fig. 4: Graphical representation of the second item in Proposition 2. In this figure, $A(x)$ and $A^{c}(x)$ represent the pairs $\left(\mu_{A}(x), \nu_{A}(x)\right)$ and $\left(\nu_{A}(x), \mu_{A}(x)\right)$, respectively.

So far we have investigated the properties of the closest crisp set to an AIFS. Now, we use this notion to define an SK-entropy in terms of local AIF-divergences. Recall that the aim of an SK-entropy is to measure how different is an AIFS from a crisp set. Therefore, it seems reasonable to measure the entropy of an AIFS as the AIF-divergence between such AIFS and its closest crisp set. Note that, when comparing $A \in \operatorname{AIFS}(X)$ with $C_{A}$ by means of a local AIF-divergence $D$ induced by the function $h_{\mathrm{IF}}$, the domain of $h_{\mathrm{IF}}$ is no longer $\mathcal{D}$, but

$$
\begin{aligned}
\mathcal{D}_{1}=\{(x, y, 1,0) \in \mathcal{D} \mid & x \geq y\} \\
& \cup\{(x, y, 0,1)) \in \mathcal{D} \mid x<y\} .
\end{aligned}
$$

For this, only the conditions imposed on $h_{\mathrm{IF}}$ in the next theorem need to be satisfied in the domain $\mathcal{D}_{1}$.

Theorem 3 Consider a local AIF-divergence $D$ induced by a function $h_{\mathrm{IF}}$, and define $E: \operatorname{AIFS}(X) \rightarrow[0,1]$ by:

$$
\begin{aligned}
E(A) & =D\left(A, C_{A}\right) \\
& =\frac{1}{n} \sum_{x \in X} h_{\mathrm{IF}}\left(\mu_{A}(x), \nu_{A}(x), \mu_{C_{A}}(x), \nu_{C_{A}}(x)\right)
\end{aligned}
$$

for any $A \subseteq X$. Then, $E$ is an $S K$-entropy if and only if function $h_{\mathrm{IF}}$ satisfies the following additional properties on $\mathcal{D}_{1}$ :

(AIF-loc.1') $h_{\mathrm{IF}}\left(u_{1}, u_{2}, v_{1}, v_{2}\right)=0$ for $\left(u_{1}, u_{2}, v_{1}, v_{2}\right) \in$ $\mathcal{D}_{1}$ if and only if $u_{1}=v_{1}, u_{2}=v_{2}$.

(AIF-loc.5) $h_{\mathrm{IF}}\left(u_{1}, u_{2}, v_{1}, v_{2}\right)=h_{\mathrm{IF}}\left(u_{2}, u_{1}, v_{2}, v_{1}\right)$ for any $\left(u_{1}, u_{2}, v_{1}, v_{2}\right) \in \mathcal{D}_{1}$ such that $u_{1} \neq u_{2}$.

(AIF-loc.6) $h_{\mathrm{IF}}\left(u_{1}, u_{2}, v_{1}, v_{2}\right)=1$ for $\left(u_{1}, u_{2}, v_{1}, v_{2}\right) \in$

$\mathcal{D}_{1}$ if and only if $u_{1}=u_{2}=0$ and $v_{1}=1, v_{2}=0$.

Proof Let us first prove that if $h_{\mathrm{IF}}$ satisfies these additional properties, then $E$ is an SK-entropy.

$\left(\mathbf{I}_{\mathbf{S K}} \mathbf{1}\right): E(A)=0$ if and only if for any $x \in X$ :

$h_{\mathrm{IF}}\left(\mu_{A}(x), \nu_{A}(x), \mu_{C_{A}}(x), \nu_{C_{A}}(x)\right)=0$. 
According to (AIF-loc. $1^{\prime}$ ), this is equivalent to $\mu_{A}(x)=$ $\mu_{C_{A}}(x)$ and $\nu_{A}(x)=\nu_{C_{A}}(x)$, which from Proposition 2 happens if and only if $A$ is a crisp set.

$\left(\mathbf{I}_{\mathbf{S K}} \mathbf{2}^{\prime}\right): E(A)=1$ if and only if

$h_{\mathrm{IF}}\left(\mu_{A}(x), \nu_{A}(x), \mu_{C_{A}}(x), \nu_{C_{A}}(x)\right)=1$

for any $x \in X$. From (AIF-loc.6), this happens if and only if $\left(u_{1}, u_{2}, v_{1}, v_{2}\right)=(0,0,1,0)$, which is equivalent to $\mu_{A}(x)=0$ and $\nu_{A}(x)=0$, that is, if and only if $\pi_{A}(x)=1$ for any $x \in X$.

$\left(\mathbf{I}_{\mathbf{S K}} \mathbf{3}\right)$ : In order to check that $E(A)=E\left(A^{c}\right)$, it is enough to check whether the following equality holds for any $x \in X$ :

$$
\begin{aligned}
& h_{\mathrm{IF}}\left(\mu_{A}(x), \nu_{A}(x), \mu_{C_{A}}(x), \nu_{C_{A}}(x)\right) \\
& \quad=h_{\mathrm{IF}}\left(\mu_{A^{c}}(x), \nu_{A^{c}}(x), \mu_{C_{A^{c}}}(x), \nu_{C_{A^{c}}}(x)\right) .
\end{aligned}
$$

On the one hand, if $\mu_{A}(x) \neq \nu_{A}(x)$, from Proposition 2, $C_{A}^{c}(x)=C_{A^{c}}(x)$, and therefore:

$$
\begin{aligned}
h_{\mathrm{IF}}\left(\mu_{A}(x),\right. & \left.\nu_{A}(x), \mu_{C_{A}}(x), \nu_{C_{A}}(x)\right) \\
& =h_{\mathrm{IF}}\left(\nu_{A}(x), \mu_{A}(x), \nu_{C_{A}}(x), \mu_{C_{A}}(x)\right) \\
& =h_{\mathrm{IF}}\left(\mu_{A^{c}}(x), \nu_{A^{c}}(x), \mu_{C_{A^{c}}}(x), \nu_{C_{A^{c}}}(x)\right),
\end{aligned}
$$

where the first equality follows from property (AIFloc.5). On the other hand, if $\mu_{A}(x)=\nu_{A}(x)$, it trivially holds that

$h_{\mathrm{IF}}\left(\mu_{A}(x), \nu_{A}(x), 1,0\right)=h_{\mathrm{IF}}\left(\nu_{A}(x), \mu_{A}(x), 1,0\right)$.

$\left(\mathbf{I}_{\mathbf{S K}} \mathbf{4}\right)$ : Assume that $\mu_{B}(x)<\nu_{B}(x)$ and $\mu_{A}(x) \leq$ $\mu_{B}(x)<\nu_{B}(x) \leq \nu_{A}(x)$, which implies that $\mu_{C_{A}}(x)=$ $\mu_{C_{B}}(x)=0$ and $\nu_{C_{A}}(x)=\nu_{C_{B}}(x)=1$. Define the following AIFSs on $\{x\}$ by:

$A^{*}=\left\langle\left(x, \mu_{A}(x), \nu_{A}(x)\right)\right\rangle, \quad B^{*}=\left\langle\left(x, \mu_{B}(x), \nu_{B}(x)\right)\right\rangle$, $N=\langle(x, 0,1)\rangle$.

It holds that $N \subseteq A^{*} \subseteq B^{*}$, and therefore from Equation $(1), D\left(A^{*}, N\right) \leq \bar{D}\left(B^{*}, N\right)$, which implies that

$$
\begin{aligned}
h_{\mathrm{IF}}\left(\mu_{A}(x)\right. & \left., \nu_{A}(x), \mu_{C_{A}}(x), \nu_{C_{A}}(x)\right) \\
& =h_{\mathrm{IF}}\left(\mu_{A}(x), \nu_{A}(x), 1,0\right) \\
& \leq h_{\mathrm{IF}}\left(\mu_{B}(x), \nu_{B}(x), 1,0\right) \\
& =h_{\mathrm{IF}}\left(\mu_{B}(x), \nu_{B}(x), \mu_{C_{B}}(x), \nu_{C_{B}}(x)\right) .
\end{aligned}
$$

On the other hand, assume $\mu_{B}(x) \geq \nu_{B}(x)$ and $\nu_{A}(x) \leq$ $\nu_{B}(x) \leq \mu_{B}(x) \leq \mu_{A}(x)$, which implies that $\mu_{C_{A}}(x)=$ $\mu_{C_{B}}(x)=1$ and $\nu_{C_{A}}(x)=\nu_{C_{B}}(x)=0$.

Now define the following AIFSs on $\{x\}$ :

$$
\begin{aligned}
& A^{*}=\left\langle\left(x, \mu_{A}(x), \nu_{A}(x)\right)\right\rangle, \quad B^{*}=\left\langle\left(x, \mu_{B}(x), \nu_{B}(x)\right)\right\rangle \\
& M=\langle(x, 1,0)\rangle .
\end{aligned}
$$

It holds that $B^{*} \subseteq A^{*} \subseteq M$, which implies, from Equation (1), that $D\left(B^{*}, M\right) \geq D\left(A^{*}, M\right)$, and therefore

$$
\begin{aligned}
h_{\mathrm{IF}}\left(\mu_{A}(x),\right. & \left.\nu_{A}(x), \mu_{C_{A}}(x), \nu_{C_{A}}(x)\right) \\
& =h_{\mathrm{IF}}\left(\mu_{A}(x), \nu_{A}(x), 0,1\right) \\
& \leq h_{\mathrm{IF}}\left(\mu_{B}(x), \nu_{B}(x), 0,1\right) \\
& =h_{\mathrm{IF}}\left(\mu_{B}(x), \nu_{B}(x), \mu_{C_{B}}(x), \nu_{C_{B}}(x)\right) .
\end{aligned}
$$

We conclude that if $h_{\mathrm{IF}}$ satisfies the additional conditions, $E$ is an SK-entropy.

On the other hand, assume that $E$ is an SK-entropy. We need to prove that $h_{\mathrm{IF}}$ satisfies the additional conditions.

$\left(\right.$ AIF - loc. $\left.\mathbf{1}^{\prime}\right):$ Take $\left(u_{1}, u_{2}, v_{1}, v_{2}\right) \in \mathcal{D}_{1}$, and define the AIFS $A$ on $\{x\}$ by $A=\left\langle\left(x, u_{1}, u_{2}\right)\right\rangle$. Note that:

$E(A)=h_{\mathrm{IF}}\left(u_{1}, u_{2}, v_{1}, v_{2}\right)$.

Hence, from $\left(I_{\mathrm{SK}} 1\right)$, it holds that

$$
E(A)=h_{\mathrm{IF}}\left(u_{1}, u_{2}, v_{1}, v_{2}\right)=0
$$

if and only if $A$ is a crisp set, which from Proposition 2 is equivalent to $A=C_{A}$. For this equality, it must hold that either $u_{1}=1$ and $u_{2}=0$, which in turn implies that $v_{1}=1$ and $v_{2}=0$, or $u_{1}=0$ and $u_{2}=1$, which implies that $v_{1}=0$ and $v_{2}=1$. In both cases, we conclude that $h_{\mathrm{IF}}\left(u_{1}, u_{2}, v_{1}, v_{2}\right)=0$ if and only if $u_{1}=$ $v_{1}$ and $u_{2}=v_{2}$.

$\left(\right.$ AIF - loc.5) : Take $\left(u_{1}, u_{2}, v_{1}, v_{2}\right) \in \mathcal{D}_{1}$, and define the AIFS $A$ on $\{x\}$ by $A=\left\langle\left(x, u_{1}, u_{2}\right)\right\rangle$. It holds that:

$E(A)=h_{\mathrm{IF}}\left(u_{1}, u_{2}, v_{1}, v_{2}\right)$

Let us assume that $u_{1} \neq u_{2}$. This means that $A^{c}=$ $\left\langle\left(x, u_{2}, u_{1}\right)\right\rangle$ and, from the second item in Proposition 2, $C_{A}^{c}=C_{A^{c}}$. Hence:

$E\left(A^{c}\right)=h_{\mathrm{IF}}\left(u_{2}, u_{1}, v_{2}, v_{1}\right)$.

Finally, from $\left(I_{\mathrm{SK}} 3\right), E(A)=E\left(A^{c}\right)$, and we deduce that $h_{\mathrm{IF}}\left(u_{1}, u_{2}, v_{1}, v_{2}\right)=h_{\mathrm{IF}}\left(u_{2}, u_{1}, v_{2}, v_{1}\right)$.

$\left(\mathbf{A I F}-\right.$ loc.6) : Take $\left(u_{1}, u_{2}, v_{1}, v_{2}\right) \in \mathcal{D}_{1}$, and define the AIFS $A$ on $\{x\}$ by $A=\left\langle\left(x, u_{1}, u_{2}\right)\right\rangle$. Then, it holds that:

$E(A)=h_{\mathrm{IF}}\left(u_{1}, u_{2}, v_{1}, v_{2}\right)$.

Therefore, $h_{\mathrm{IF}}\left(u_{1}, u_{2}, v_{1}, v_{2}\right)=1$ if and only if $E(A)=$ 1 , which from $\left(I_{\mathrm{SK}} 2^{\prime}\right)$, is equivalent to $u_{1}=u_{2}=0$ and $v_{1}=1, v_{2}=0$.

We conclude that if $E$ is an SK-entropy, $h_{\mathrm{IF}}$ must satisfy the additional conditions. 
In [?] we have shown how an AIF-divergence could be built from a divergence for fuzzy sets. In particular, given a divergence $D$ for fuzzy sets and a componentwise increasing function $f:[0,1] \times[0,1] \rightarrow[0,1]$ satisfying $f(0,0)=0$, the function

$D_{\mathrm{AIF}}(A, B)=f\left(D\left(\mu_{A}, \mu_{B}\right), D\left(\nu_{A}, \nu_{B}\right)\right)$

for any $A, B \in \operatorname{AIFS}(X)$ is an $\mathrm{AIF}$-divergence, where $\mu_{A}, \nu_{A}, \mu_{B}, \nu_{B}$ are considered as fuzzy sets [?, Prop. 4.7]. Furthermore, if $D$ is local, $D_{\text {AIF }}$ is local if and only if $f(x, y)=\alpha x+\beta y$ for some $\alpha, \beta \geq 0$ [?, Prop. 5.2].

Following a similar reasoning, we can define an SKentropy using a local divergence for fuzzy sets, just imposing some additional conditions on $f$ and on the fuzzy divergence. For this, we consider the following domain where the function $h_{\mathrm{FS}}$ will be defined:

$\mathcal{D}_{2}=\{(x, y) \mid y=1\} \cup\left\{(x, y) \mid x \leq \frac{1}{2}, y=0\right\}$.

Proposition 3 Consider a local divergence $D$ for fuzzy sets induced by the function $h_{\mathrm{FS}}$ and let $f:[0,1] \times$ $[0,1) \rightarrow[0,1]$ be a function satisfying

(f1) $f(u, v)=0$ if and only if $u=v=0$.

(f2) $f$ is component-wise increasing.

(f3) $f(u, v)=1$ if and only if $u=1$ and $v=0$.

(f4) $f(u, v)=f(u, v)$.

Then, the function $E$ defined by:

$E(A)=\frac{1}{n} \sum_{x \in X} f\left(h_{\mathrm{FS}}\left(\mu_{A}(x), \mu_{C_{A}}(x)\right)\right.$,

$$
\left.h_{\mathrm{FS}}\left(\nu_{A}(x), \nu_{C_{A}}(x)\right)\right)
$$

is an SK-entropy if and only if $h_{\mathrm{FS}}$ satisfies the following additional conditions in $\mathcal{D}_{2}$ :

(loc.1') For $(u, v) \in \mathcal{D}_{2}, h_{\mathrm{FS}}(u, v)=0$ if and only if $u=v$;

(loc.4) For $(u, v) \in \mathcal{D}_{2}, h_{\mathrm{FS}}(u, v)=1$ if and only if $u=0, v=1$.

Proof First of all, assume that $h_{\mathrm{FS}}$ satisfies the additional conditions and let us prove that $E$ is an SKentropy.

$\left(\mathbf{I}_{\mathbf{S K}} \mathbf{1}\right): E(A)=0$ if and only if

$f\left(h_{\mathrm{FS}}\left(\mu_{A}(x), \mu_{C_{A}}(x)\right), h_{\mathrm{FS}}\left(\nu_{A}(x), \nu_{C_{A}}(x)\right)\right)=0$

for any $x \in X$. From (f1), $f(u, v)=0$ if and only if $u=v=0$, which is equivalent to

$h_{\mathrm{FS}}\left(\mu_{A}(x), \mu_{C_{A}}(x)\right)=h_{\mathrm{FS}}\left(\nu_{A}(x), \nu_{C_{A}}(x)\right)=0$,

but from (loc.1') this happens if and only if $\mu_{A}(x)=$ $\mu_{C_{A}}(x)$ and $\nu_{A}(x)=\nu_{C_{A}}(x)$, which from Proposition 2 is equivalent to $A=C_{A}$, so $A$ is a crisp set.
$\left(\mathbf{I}_{\mathbf{S K}} \mathbf{2}^{\prime}\right): E(A)=1$ if and only if $f\left(h_{\mathrm{FS}}\left(\mu_{A}(x), \mu_{C_{A}}(x)\right), h_{\mathrm{FS}}\left(\nu_{A}(x), \nu_{C_{A}}(x)\right)\right)=1$

for any $x \in X$. From (f3), this happens if and only if $h_{\mathrm{FS}}\left(\mu_{A}(x), \mu_{C_{A}}(x)\right)=1$ and $h_{\mathrm{FS}}\left(\nu_{A}(x), \nu_{C_{A}}(x)\right)=0$. Also, from (loc.4) it holds that $h_{\mathrm{FS}}(u, v)=1$ if and only if $u=0, v=1$. However, this happens if and only if $\mu_{A}(x)=0$ and $\mu_{C_{A}}(x)=1$, which is equivalent to $\nu_{A}(x)=0$.

$\left(\mathbf{I}_{\mathbf{S K}} \mathbf{3}\right)$ : In order to prove $E(A)=E\left(A^{c}\right)$, we will see that:

$$
\begin{aligned}
& f\left(h_{\mathrm{FS}}\left(\mu_{A}(x), \mu_{C_{A}}(x)\right), h_{\mathrm{FS}}\left(\nu_{A}(x), \nu_{C_{A}}(x)\right)\right) \\
& \quad=f\left(h_{\mathrm{FS}}\left(\mu_{A^{c}}(x), \mu_{C_{A^{c}}}(x)\right), h_{\mathrm{FS}}\left(\nu_{A^{c}}(x), \nu_{C_{A^{c}}}(x)\right)\right)
\end{aligned}
$$

for any $x \in X$. First of all, since $\mu_{A^{c}}(x)=\nu_{A}(x)$ and $\nu_{A^{c}}(x)=\mu_{A}(x)$, we only need to prove that:

$$
\begin{aligned}
& f\left(h_{\mathrm{FS}}\left(\mu_{A}(x), \mu_{C_{A}}(x)\right), h_{\mathrm{FS}}\left(\nu_{A}(x), \nu_{C_{A}}(x)\right)\right) \\
& \quad=f\left(h_{\mathrm{FS}}\left(\nu_{A}(x), \mu_{C_{A^{c}}}(x)\right), h_{\mathrm{FS}}\left(\mu_{A}(x), \nu_{C_{A^{c}}}(x)\right)\right) .
\end{aligned}
$$

If $\mu_{A}(x)=\nu_{A}(x)$, this means that $\mu_{C_{A}}(x)=\mu_{C_{A^{c}}}(x)=$ 1 and $\nu_{C_{A}}(x)=\nu_{C_{A^{c}}}(x)=0$, which implies that the equality in Equation (6) holds.

Assume now that $\mu_{A}(x) \neq \nu_{A}(x)$. From Proposition $2, C_{A}^{c}(x)=C_{A^{c}}(x)$, which means that $\mu_{C_{A}}(x)=$ $\nu_{C_{A^{c}}}(x)$ and $\nu_{C_{A}}(x)=\mu_{C_{A^{c}}}(x)$. Also, note that $\mu_{A}(x)=$ $\nu_{A^{c}}(x)$ and $\nu_{A}(x)=\mu_{A^{c}}(x)$. Using these facts, as well as property (f4), it holds that:

$$
\begin{aligned}
f( & \left.h_{\mathrm{FS}}\left(\mu_{A}(x), \mu_{C_{A}}(x)\right), h_{\mathrm{FS}}\left(\nu_{A}(x), \nu_{C_{A}}(x)\right)\right) \\
& =f\left(h_{\mathrm{FS}}\left(\nu_{A^{c}}(x), \nu_{C_{A^{c}}}(x)\right), h_{\mathrm{FS}}\left(\mu_{A^{c}}(x), \mu_{C_{A^{c}}}(x)\right)\right) \\
& =f\left(h_{\mathrm{FS}}\left(\mu_{A^{c}}(x), \mu_{C_{A^{c}}}(x)\right), h_{\mathrm{FS}}\left(\nu_{A^{c}}(x), \nu_{C_{A^{c}}}(x)\right)\right) \\
& =f\left(h_{\mathrm{FS}}\left(\nu_{A}(x), \mu_{C_{A^{c}}}(x)\right), h_{\mathrm{FS}}\left(\mu_{A}(x), \nu_{C_{A^{c}}}(x)\right)\right) .
\end{aligned}
$$

$\left(\mathbf{I}_{\mathbf{S K}} 4\right)$ : Assume that $\mu_{A}(x) \leq \mu_{B}(x)<\nu_{B}(x) \leq$ $\nu_{A}(x)$. In this case, it holds that $\mu_{C_{A}}(x)=\mu_{C_{B}}(x)=0$ and $\nu_{C_{A}}(x)=\nu_{C_{B}}(x)=1$. By the property (loc.3) of $h_{\mathrm{FS}}$, it follows that:

$h_{\mathrm{FS}}\left(\mu_{A}(x), 0\right) \leq h_{\mathrm{FS}}\left(\mu_{B}(x), 0\right)$ and

$h_{\mathrm{FS}}\left(\nu_{A}(x), 0\right) \leq h_{\mathrm{FS}}\left(\nu_{B}(x), 0\right)$,

and by (f2), it follows that

$$
\begin{aligned}
f\left(h_{\mathrm{FS}}\right. & \left.\left(\mu_{A}(x), \mu_{C_{A}}(x)\right), h_{\mathrm{FS}}\left(\nu_{A}(x), \nu_{C_{A}}(x)\right)\right) \\
& =f\left(h_{\mathrm{FS}}\left(\mu_{A}(x), 0\right), h_{\mathrm{FS}}\left(\nu_{A}(x), 1\right)\right) \\
& \leq f\left(h_{\mathrm{FS}}\left(\mu_{B}(x), 0\right), h_{\mathrm{FS}}\left(\nu_{B}(x), 1\right)\right) \\
& =f\left(h_{\mathrm{FS}}\left(\mu_{B}(x), \mu_{C_{B}}(x)\right), h_{\mathrm{FS}}\left(\nu_{B}(x), \nu_{C_{B}}(x)\right)\right) .
\end{aligned}
$$

Assume now that $\nu_{A}(x) \leq \nu_{B}(x) \leq \mu_{B}(x) \leq \mu_{A}(x)$, which implies that $\mu_{C_{A}}(x)=\mu_{C_{B}}(x)=1$ and $\nu_{C_{A}}(x)=$ 
$\nu_{C_{B}}(x)=0$. Using the property (loc.3) of $h_{\mathrm{FS}}$, it holds that:

$h_{\mathrm{FS}}\left(\mu_{A}(x), 1\right) \leq h_{\mathrm{FS}}\left(\mu_{B}(x), 1\right)$ and

$h_{\mathrm{FS}}\left(\nu_{A}(x), 0\right) \leq h_{\mathrm{FS}}\left(\nu_{B}(x), 0\right)$.

Also, (f2) implies that:

$$
\begin{aligned}
f\left(h_{\mathrm{FS}}\right. & \left.\left(\mu_{A}(x), \mu_{C_{A}}(x)\right), h_{\mathrm{FS}}\left(\nu_{A}(x), \nu_{C_{A}}(x)\right)\right) \\
& =f\left(h_{\mathrm{FS}}\left(\mu_{A}(x), 1\right), h_{\mathrm{FS}}\left(\nu_{A}(x), 0\right)\right) \\
& \leq f\left(h_{\mathrm{FS}}\left(\mu_{B}(x), 1\right), h_{\mathrm{FS}}\left(\nu_{B}(x), 0\right)\right) \\
& =f\left(h_{\mathrm{FS}}\left(\mu_{B}(x), \mu_{C_{B}}(x)\right), h_{\mathrm{FS}}\left(\nu_{B}(x), \nu_{C_{B}}(x)\right)\right) .
\end{aligned}
$$

On the other hand, let us now assume that $E$ is an SKentropy and we prove that $f$ and $h_{\mathrm{FS}}$ must satisfy the additional conditions.

(loc.1') : On the one hand, take $(u, v) \in \mathcal{D}_{2}$ such that $v=1$, and define the AIFS $A$ on $\{x\}$ by $A=$ $\langle(x, u, 0)\rangle$. Then, $C_{A}(x)=1$, hence:

$E(A)=f\left(h_{\mathrm{FS}}(u, 1), h_{\mathrm{FS}}(0,0)\right)$.

Also, from ( $\left.I_{\mathbf{S K}} 1\right), E(A)=0$ if and only if $A$ is a crisp set, which by Proposition 2 is equivalent to $A=C_{A}$. This holds if and only if $u=1$. Finally, $E(A)=0$ is equivalent to:

$f\left(h_{\mathrm{FS}}(u, 1), h_{\mathrm{FS}}(0,0)\right)=0$,

but from (f1) this happens if and only if $h_{\mathrm{FS}}(u, 1)=$ $h_{\mathrm{FS}}(0,0)=0$. We therefore conclude that $u=1$ if and only if $h_{\mathrm{FS}}(u, 1)=0$.

On the other hand, take $(u, v) \in \mathcal{D}_{2}$ such that $v=0$ and define the AIFS $A$ on $\{x\}$ such that $A=\langle(x, 1-$ $u, u)\rangle$. Note that since $(u, 0) \in \mathcal{D}_{2}$, this implies that $u \leq \frac{1}{2}$, or equivalently, $1-u \geq \frac{1}{2}$. Then, $C_{A}(x)=1$, hence:

$E(A)=f\left(h_{\mathrm{FS}}(1-u, 1), h_{\mathrm{FS}}(u, 0)\right)$.

Now, from $\left(I_{\mathbf{S K}} 1\right), E(A)=0$ if and only if $A$ is a crisp set, which by Proposition 2 is equivalent to $A=C_{A}$. This happens if and only if $u=0$. Finally, $E(A)=0$ is equivalent to

$f\left(h_{\mathrm{FS}}(1-u, 1), h_{\mathrm{FS}}(u, 0)\right)=0$,

but from (f1) this happens if and only if $h_{\mathrm{FS}}(1-u, 1)=$ $h_{\mathrm{FS}}(u, 0)=0$. We conclude that $u=0$ if and only if $h_{\mathrm{FS}}(u, 0)=0$.

(loc.4) : First of all, take $(u, v)=(0,1) \in \mathcal{D}_{2}$ and define the AIFS $A$ on $\{x\}$ by $A=\langle(x, 0,0)\rangle$. From $\left(I_{\mathbf{S K}} 2^{\prime}\right)$ :

$E(A)=f\left(h_{\mathrm{FS}}(0,1), h_{\mathrm{FS}}(0,0)\right)=1$.

However, using (f3), this happens if and only if $h_{\mathrm{FS}}(0,1)=$ 1 and $h_{\mathrm{FS}}(0,0)=0$. We conclude that $h_{\mathrm{FS}}(0,1)=1$.
On the other hand, let us see that if $h_{\mathrm{FS}}(u, v)=1$, it must hold that $u=0, v=1$. First of all, assume that $(u, v) \in \mathcal{D}_{2}$ such that $v=0$ and $h_{\mathrm{FS}}(u, v)=1$. This means that $u<1-u$. Let us define the AIFS $A$ on $\{x\}$ by $A=\langle(x, u, 1-u)\rangle$. Using the definition of $E$ and (f2), it holds that:

$$
\begin{aligned}
E(A) & =f\left(h_{\mathrm{FS}}(u, 0), h_{\mathrm{FS}}(1-u, 1)\right) \\
& =f\left(1, h_{\mathrm{FS}}(1-u, 1)\right) \geq f(1,0)=1,
\end{aligned}
$$

where the last equality follows from (f3). We conclude that $E(A)=1$. However, from $\left(I_{\mathbf{S K}} 2^{\prime}\right)$ this is equivalent to $u=1-u=0$, a contradiction.

Therefore, take $(u, v) \in \mathcal{D}_{2}$ such that $v=1$, and define the AIFS $A$ on $\{x\}$ by $A=\langle(x, u, 0)\rangle$. Then, it holds that:

$$
E(A)=f\left(h_{\mathrm{FS}}(u, 1), h_{\mathrm{FS}}(0,0)\right)=f\left(h_{\mathrm{FS}}(u, 1), 0\right),
$$

where the last equality follows from (loc.1). Now, from (f3), $f\left(h_{\mathrm{FS}}(u, 1), 0\right)=1$ if and only if $h_{\mathrm{FS}}(u, 1)=1$. However, from $\left(I_{\mathbf{S K}} 2^{\prime}\right), E(A)=1$ if and only if $u=0$. Therefore, we conclude that $u=0$ and $h_{\mathrm{FS}}(u, 1)=1$ are equivalent when $v=1$.

Remark 1 Note that in the previous result, the domain of the function $f$ is $[0,1] \times[0,1)$. The reason for not including the value 1 in the second component is that $h_{\mathrm{FS}}\left(\nu_{A}(x), \nu_{C_{A}}(x)\right)$ cannot take the value 1 : according to (loc. 4$), h_{\mathrm{FS}}(u, v)=1$ if and only if $u=0, v=1$, but this would mean that $\nu_{A}(x)=0$ and $\nu_{C_{A}}(x)=1$, a contradiction, because $\nu_{A}(x)=0$ implies that $\mu_{A}(x) \geq$ $\nu_{A}(x)$ and therefore $\nu_{C_{A}}(x)=0$.

\subsection{Examples of SK-entropies based on AIF-divergences}

In the literature, several different measures of comparison have been introduced. In an earlier work [?, Section III-C] we showed some examples of local AIF-divergences, like the Hamming [?] and Hausdorff [?] distances, denoted by $l_{A I F}$ and $d_{H}$, respectively, and the two measures proposed by Hong \& Kim [?] $]^{2}$, denoted by $D_{C}$ and $D_{L}$. In this subsection we consider these four local AIF-divergences and we investigate whether they satisfy the conditions of Theorem 3 and can therefore be used to define a SK-entropy. Recall that these

2 The original definition of $D_{C}$ and $D_{L}$ is slightly different from those of Equations (9) and (10). The difference is that in [?], $D_{C}$ was divided by 2 and $D_{L}$ by 4 , instead of 2 . In this paper, we consider the definitions of Equations (9) and (10) just to make $D_{C}$ and $D_{L}$ to satisfy the normalization property mentioned in Subsection 2.3. 
AIF-divergences are defined by:

$$
\begin{aligned}
& l_{A I F S}(A, B)=\frac{1}{2 n} \sum_{x \in X}\left|\mu_{A}(x)-\mu_{B}(x)\right| \\
& \quad+\left|\nu_{A}(x)-\nu_{B}(x)\right|+\left|\pi_{A}(x)-\pi_{B}(x)\right| \\
& d_{H}(A, B)= \\
& \frac{1}{n} \sum_{x \in X} \max \left\{\left|\mu_{A}(x)-\mu_{B}(x)\right|,\left|\nu_{A}(x)-\nu_{B}(x)\right|\right\} \\
& \quad+\left|\nu_{A}(x)-\nu_{B}(x)\right| . \\
& D_{C}(A, B)=\frac{1}{n} \sum_{x \in X}\left|\mu_{A}(x)-\mu_{B}(x)\right| \\
& \quad+\left|\mu_{A}(x)-\mu_{B}(x)\right|+\left|\nu_{A}(x)-\nu_{B}(x)\right| .
\end{aligned}
$$

In the next example we show that both the Hamming and Hausdorff distances satisfy the conditions of Theorem 3 and, surprisingly, they both induce the same SK-entropy. On the contrary, we show that the Hong and Kim divergences do not induce a SK-entropy.

Example 1 Consider first the Hamming and Hausdorff distances defined in Equations (7) and (8), and let us see that their associated functions, that will be denoted by $h_{l}$ and $h_{d}$, satisfy the conditions of Theorem 3 . First of all, note that the functions $h_{l}$ and $h_{d}$ inducing the Hamming and Hausdorff distances are given by:

$$
\begin{aligned}
h_{l}\left(u_{1}, u_{2}, v_{1}, v_{2}\right)= & \frac{1}{2}\left(\left|u_{1}-v_{1}\right|+\left|u_{2}-v_{2}\right|\right. \\
& \left.+\left|u_{1}+u_{2}-v_{1}-v_{2}\right|\right) . \\
h_{d}\left(u_{1}, u_{2}, v_{1}, v_{2}\right)= & \max \left\{\left|u_{1}-v_{1}\right|,\left|u_{2}-v_{2}\right|\right\} .
\end{aligned}
$$

Also:

$$
\begin{aligned}
& h_{l}(u, v, 1,0)=\frac{1}{2}((1-u)+v+(1-u-v))=1-u . \\
& h_{l}(u, v, 0,1)=\frac{1}{2}(u+(1-v)+(1-u-v))=1-v . \\
& h_{d}(u, v, 1,0)=\max \{1-u, v\}=1-u . \\
& h_{d}(u, v, 0,1)=\max \{u, 1-v\}=1-v .
\end{aligned}
$$

Therefore, we can see that $h_{l}$ and $h_{d}$ coincide in $\mathcal{D}_{1}$. Now, let us prove that $h_{l}$, and consequently also $h_{d}$, satisfies the required properties:

$\left(\mathbf{A I F}-\right.$ loc.1 $\left.^{\prime}\right) h_{l}(u, v, 1,0)=0$ if and only if $1-$ $u=0$, or equivalently, if and only if $u=1$. But also, $u=$ 1 is equivalent to $v=0$, because $u+v \leq 1$. Similarly, $h_{l}(u, v, 0,1)=0$ if and only if $1-v=0$, or equivalently, if and only if $v=1$. But this is equivalent to $u=0$ because $u+v \leq 1$.

(AIF - loc.5) In [?, Section IV], we have proven that $h_{l}$ is symmetric.
(AIF - loc.6) Finally, $h_{l}(u, v, 1,0)=1$ if and only if $u=0$, but this can only happen if and only if $v=0$. On the other hand, $h_{l}(u, v, 0,1)=1$ is not possible, because this would mean that $v=0$, but in that case we would compare $(u, v)$ with $(1,0)$, not with $(0,1)$.

We conclude that both $h_{l}$ and $h_{d}$ satisfy the additional conditions of Theorem 3 , so each of $l_{A I F}$ and $d_{H}$ induces an SK-entropy. Furthermore, since $h_{l}=h_{d}$ in the domain $\mathcal{D}_{1}$ we are considering in the definition of the entropy, we conclude that they induce the same SK-entropy.

Once that we know that they both induce a SKentropy, let us see that the SK-entropy they induce coincide and it is given by:

$E(A)=1-\frac{1}{n} \sum_{x \in X} \max \left\{\mu_{A}(x), \nu_{A}(x)\right\}$.

On the one hand, if $\mu_{A}(x) \geq \nu_{A}(x), \mu_{C_{A}}(x)=1, \nu_{C_{A}}(x)=$ 0 so:

$$
\begin{aligned}
h_{l}\left(\mu_{A}(x), \nu_{A}(x), \mu_{C_{A}}(x), \nu_{C_{A}}(x)\right) \\
\quad=h_{l}\left(\mu_{A}(x), \nu_{A}(x), 1,0\right)=1-\mu_{A}(x) .
\end{aligned}
$$

On the other hand, if $\mu_{A}(x)<\nu_{A}(x), \mu_{C_{A}}(x)=0$, $\nu_{C_{A}}(x)=1$, and then:

$$
\begin{aligned}
h_{l}\left(\mu_{A}(x), \nu_{A}(x), \mu_{C_{A}}(x), \nu_{C_{A}}(x)\right) \\
=h_{l}\left(\mu_{A}(x), \nu_{A}(x), 0,1\right)=1-\nu_{A}(x) .
\end{aligned}
$$

Substituting this values, we obtain the following:

$$
\begin{aligned}
& E(A)=\frac{1}{n}\left(\sum_{x \mid \mu_{A}(x) \geq \nu_{A}(x)} 1-\mu_{A}(x)\right.\left.+\sum_{x \mid \mu_{A}(x)<\nu_{A}(x)} 1-\nu_{A}(x)\right) \\
&=1-\frac{1}{n}\left(\sum_{x \mid \mu_{A}(x) \geq \nu_{A}(x)} \mu_{A}(x)\right.\left.+\sum_{x \mid \mu_{A}(x)<\nu_{A}(x)} \nu_{A}(x)\right) \\
&=1-\frac{1}{n} \sum_{x \in X} \max \left\{\mu_{A}(x), \nu_{A}(x)\right\} . \square
\end{aligned}
$$

Let us now see that the local AIF-divergences of Hong and Kim defined in Equations (9) and (10) do not satisfy the conditions of Theorem 3 . It can be easily seen that the functions $h_{C}$ and $h_{L}$ associated with $D_{C}$ 
and $D_{L}$, respectively, are given by:

$$
\begin{aligned}
h_{C}\left(u_{1}, u_{2}, v_{1}, v_{2}\right) & =\left|u_{1}-v_{1}\right|+\left|u_{2}-v_{2}\right| . \\
h_{L}\left(u_{1}, u_{2}, v_{1}, v_{2}\right)= & \frac{1}{2}\left(\left|u_{1}-u_{2}-v_{1}+v_{2}\right|\right. \\
& \left.\quad+\left|u_{1}-v_{1}\right|+\left|u_{2}-v_{2}\right|\right) .
\end{aligned}
$$

However, these functions do not satisfy one of the conditions of Theorem 3 because for $\alpha=\mu_{A}(x)=\nu_{A}(x)>0$, it happens that $\mu_{C_{A}}(x)=1, \nu_{C_{A}}(x)=0$ and:

$h_{C}\left(\mu_{A}(x), \nu_{A}(x), \mu_{C_{A}}(x), \nu_{C_{A}}(x)\right)=h_{C}(\alpha, \alpha, 1,0)=1$,

but $\alpha \neq 0$. Therefore, neither $h_{C}$ nor $h_{D}$ satisfies (AIFloc.6), and then we conclude that neither $D_{C}$ nor $D_{L}$ induces an SK-entropy.

Next example shows that the SK-entropy defined by Guo and Song [?] by:

$$
\begin{aligned}
E(A)=\frac{1}{n} \sum_{x \in X} & \left(1-\mid \mu_{A}(x)\right. \\
& \left.-\nu_{A}(x) \mid\right) \cdot\left(\frac{2-\mu_{A}(x)-\nu_{A}(x)}{2}\right)
\end{aligned}
$$

can also be defined as in Theorem 3 through a local AIF-divergence.

Example 2 Consider now the function $D$ given for any $A, B \in \operatorname{AIFS}(X)$ by:

$$
\begin{aligned}
D(A, B) & =\frac{1}{n} \sum_{x \in X}\left(\left|\mu_{A}(x)-\mu_{B}(x)\right|+\right. \\
& \left.\left|\nu_{A}(x)-\nu_{B}(x)\right|\right) \cdot\left(\frac{1+\left|\pi_{A}(x)-\pi_{B}(x)\right|}{2}\right) .
\end{aligned}
$$

It can be easily proven that this function is a local AIFdivergence with associated function:

$$
\begin{aligned}
h_{\mathrm{IF}}\left(u_{1}, u_{2}, v_{1}, v_{2}\right) & =\left(\left|u_{1}-v_{1}\right|\right. \\
& \left.+\left|u_{2}-v_{2}\right|\right) \cdot\left(\frac{1+\left|u_{1}+u_{2}-v_{1}-v_{2}\right|}{2}\right) .
\end{aligned}
$$

The function $h_{\mathrm{IF}}$ also satisfies the additional conditions of Theorem 3, hence it induces an SK-entropy. Note that when $u \geq v$, we obtain:

$h_{\mathrm{IF}}(u, v, 1,0)=(1-u+v) \cdot\left(\frac{2-u-v}{2}\right)$,

while for $u<v$, we obtain:

$h_{\mathrm{IF}}(u, v, 0,1)=(1-v+u) \cdot\left(\frac{2-u-v}{2}\right)$.
Therefore, the SK-entropy induced by $D$ is given by:

$$
\begin{aligned}
E(A)= & \frac{1}{n}\left(\sum _ { x | \mu _ { A } ( x ) \geq \nu _ { A } ( x ) } \left(1-\mu_{A}(x)\right.\right. \\
& \left.\quad+\nu_{A}(x)\right) \cdot\left(\frac{2-\mu_{A}(x)-\nu_{A}(x)}{2}\right) \\
& +\sum_{x \mid \mu_{A}(x)<\nu_{A}(x)}\left(1+\mu_{A}(x)\right. \\
& \left.\left.\quad-\nu_{A}(x)\right) \cdot\left(\frac{2-\mu_{A}(x)-\nu_{A}(x)}{2}\right)\right) \\
& \frac{1}{n} \sum_{x \in X}\left(1-\mid \mu_{A}(x)\right. \\
& \left.-\nu_{A}(x) \mid\right) \cdot\left(\frac{2-\mu_{A}(x)-\nu_{A}(x)}{2}\right) .
\end{aligned}
$$

To conclude the section, let us see two examples of SKentropies built using the procedure of Proposition 3.

Example 3 Consider the Hamming distance for fuzzy sets, which is defined for any $A, B \in \operatorname{AIFS}(X)$ by:

$l_{F S}(A, B)=\frac{1}{n} \sum_{x \in X}\left|\mu_{A}(x)-\mu_{B}(x)\right|$,

where $\mu_{A}$ and $\mu_{B}$ denote the membership function of two fuzzy sets $A$ and $B$. The Hamming distance for fuzzy sets is known to be a local divergence for fuzzy sets, and its associated function $h$ is given by $h(u, v)=$ $|u-v|$. This function $h$ satisfies the additional conditions required in Proposition 3. On the one hand, $h(u, v)=0$ if and only if $u=v=0$; on the other hand, $h(u, v)=|u-v|=1$ if and only if either $u=1, v=0$ or $u=0, v=1$. However, $(u, v)=(1,0) \notin \mathcal{D}_{2}$. Hence, $h(u, v)=1$ for $(u, v) \in \mathcal{D}_{2}$ if and only if $u=0, v=1$. Also, we consider the function $f$ given by $f(u, v)=$ $u+v-u v$, which is usually called the product t-conorm (see [?] for details in t-norms and t-conorms). As any t-conorm, $f$ satisfies conditions (f1), (f2) and (f4). Also, since $f$ is the t-conorm associated with a strict t-norm (algebraic product), it also satisfies (f3) ${ }^{3}$.

Therefore, applying Proposition 3 we can define an SK-entropy by using Equation (5). Note that, if $\mu_{A}(x) \geq$ $\nu_{A}(x), \mu_{C_{A}}(x)=1, \nu_{C_{A}}(x)=0$, and it holds that:

$$
\begin{aligned}
f\left(h\left(\mu_{A}(x), \mu_{C_{A}}(x)\right), h\left(\nu_{A}(x), \nu_{C_{A}}(x)\right)\right) \\
=f\left(h\left(\mu_{A}(x), 1\right), h\left(\nu_{A}(x), 0\right)\right) \\
=f\left(1-\mu_{A}(x), \nu_{A}(x)\right) \\
=1-\mu_{A}(x)+\nu_{A}(x)-\left(1-\mu_{A}(x)\right) \nu_{A}(x) \\
=\left(1-\mu_{A}(x)\right)\left(1-\nu_{A}(x)\right)+\nu_{A}(x) .
\end{aligned}
$$

3 Recall that any t-conorm $f$ satisfies $f(u, v)=1$ if and only if either $u=1$ or $v=1$. However, remember that in the statement of Proposition 3 we are restricting the domain of $f$ to the set $[0,1] \times[0,1)$, hence $f(u, v)=1$ if and only if $u=1$. 
Also, if $\mu_{A}(x)<\nu_{A}(x), \mu_{C_{A}}(x)=0, \nu_{C_{A}}(x)=1$, and then:

$$
\begin{aligned}
f\left(h \left(\mu_{A}(x),\right.\right. & \left.\left.\mu_{C_{A}}(x)\right), h\left(\nu_{A}(x), \nu_{C_{A}}(x)\right)\right) \\
& =f\left(h\left(\mu_{A}(x), 0\right), h\left(\nu_{A}(x), 1\right)\right) \\
& =f\left(\mu_{A}(x), 1-\nu_{A}(x)\right) \\
& =1-\nu_{A}(x)+\mu_{A}(x)-\left(1-\nu_{A}(x)\right) \mu_{A}(x) \\
& =\left(1-\mu_{A}(x)\right)\left(1-\nu_{A}(x)\right)+\mu_{A}(x) .
\end{aligned}
$$

Therefore, the SK-entropy defined using Equation (5) is given by:

$$
E(A)
$$

$$
\begin{aligned}
& =\frac{1}{n}\left(\sum_{x \mid \mu_{A}(x) \geq \nu_{A}(x)}\left(1-\mu_{A}(x)\right)\left(1-\nu_{A}(x)\right)+\nu_{A}(x)\right. \\
& \left.\quad+\sum_{x \mid \mu_{A}(x)<\nu_{A}(x)}\left(1-\mu_{A}(x)\right)\left(1-\nu_{A}(x)\right)+\mu_{A}(x)\right) \\
& =\frac{1}{n} \sum_{x \in X}\left(1-\mu_{A}(x)\right)\left(1-\nu_{A}(x)\right)+\min \left\{\mu_{A}(x), \nu_{A}(x)\right\} .
\end{aligned}
$$

Example 4 Consider again the Hamming distance for fuzzy sets defined on Equation (12) and take now the function $f$ given by $f(u, v)=\max \{u, v\}$. This function is also a t-conorm satisfying (f1) to (f4), so using the Hamming distance for fuzzy sets and the maximum tconorm, we can apply Proposition 3 to define an SKentropy. Let us note that for $\mu_{A}(x) \geq \nu_{A}(x), \mu_{C_{A}}(x)=$ $1, \nu_{C_{A}}(x)=0$, so:

$$
\begin{aligned}
f\left(h \left(\mu_{A}(x),\right.\right. & \left.\left.\mu_{C_{A}}(x)\right), h\left(\nu_{A}(x), \nu_{C_{A}}(x)\right)\right) \\
& =f\left(h\left(\mu_{A}(x), 1\right), h\left(\nu_{A}(x), 0\right)\right) \\
& =\max \left\{1-\mu_{A}(x), \nu_{A}(x)\right\}=1-\mu_{A}(x),
\end{aligned}
$$

and if $\mu_{A}(x)<\nu_{A}(x), \mu_{C_{A}}(x)=0, \nu_{C_{A}}(x)=1$, therefore:

$$
\begin{aligned}
f\left(h \left(\mu_{A}(x),\right.\right. & \left.\left.\mu_{C_{A}}(x)\right), h\left(\nu_{A}(x), \nu_{C_{A}}(x)\right)\right) \\
& =f\left(h\left(\mu_{A}(x), 0\right), h\left(\nu_{A}(x), 1\right)\right) \\
& =\max \left\{\mu_{A}(x), 1-\nu_{A}(x)\right\}=1-\nu_{A}(x) .
\end{aligned}
$$

However, taking Example 1 into account, the SK-entropy that we can define using the Hamming distance for fuzzy sets and the maximum t-conorm coincides with the SK-entropy defined from the Hamming and Hausdorff distances for AIFSs, given in Equation (11).

\section{BB-entropies}

We now investigate other type of entropies, those measuring how different is an AIFS from being a fuzzy set. For this aim we consider the definition of entropy given by Burrillo and Bustince in [?], and we investigate whether we can define an entropy using local AIFdivergences.

\subsection{Burrillo and Bustince's entropy}

To the best of our knowledge, the first proposal of entropy for AIFSs was introduced by Burrillo and Bustince in [?].

Definition 5 ([?]) A mapping $I: \operatorname{AIFS}(X) \rightarrow[0,1]$ is called entropy if it satisfies the following properties:

$\left(I_{\mathrm{BB}} 1\right) I(A)=0$ if and only if $A \in \mathrm{FS}(X)$.

$\left(I_{\mathrm{BB}} 2\right) I(A)=1$ if and only if $\mu_{A}=\nu_{A}=0$.

$\left(I_{\mathrm{BB}} 3\right) I(A)=I\left(A^{c}\right)$.

$\left(I_{\mathrm{BB}} 4\right) I(A) \geq I(B)$ if $\mu_{A} \leq \mu_{B}$ and $\nu_{A} \leq \nu_{B}$.

This type of entropies measures how intuitionistic is an AIF, or in other words, how different is an AIF from a fuzzy set. The first property $\left(I_{\mathrm{BB}} 1\right)$ says that the entropy is zero if, and only if, the hesitation index is zero, or equivalently, if and only if the AIFS is a fuzzy set. $\left(I_{\mathrm{BB}} 2\right)$ says that the entropy is maximal if and only if the hesitation index is 1 , which means that there is a total lack of information. The third condition says that the entropy is closed under complementaries, while $\left(I_{\mathrm{BB}} 4\right)$ means that the greater the hesitation index, the greater the entropy.

In what follows, a function $I$ satisfying properties $\left(I_{\mathrm{BB}} 1\right)$ to $\left(I_{\mathrm{BB}} 4\right)$ will be called a BB-entropy.

\subsection{BB-entropies based on local AIF-divergences}

Our aim is now to define BB-entropies using local AIFdivergences, in a similar manner as we did in Subsection 3.2. For this, we define closest fuzzy set to an AIFS.

Definition 6 Given $A \in \operatorname{AIFS}(X)$, we define the closest fuzzy set to $A$, denoted by $A^{*}$, by $\mu_{A^{*}}(x)=\mu_{A}(x)+$ $\frac{\pi_{A}(x)}{2}$.

It can be easily seen that $1-\mu_{A^{*}}(x)=\nu_{A}(x)+\frac{\pi_{A}(x)}{2}$, and the interpretation of $A^{*}$ can be seen in Figure 5 . In this figure, the fuzzy sets are those elements that belong to the line from $(1,0)$ to $(0,1)$. In order to define the closest fuzzy set to an AIFS, we find the point of the shortest distance of the point $\left(\mu_{A}(x), \nu_{A}(x)\right)$ to the $(0,1)-(1,0)$ line. This results in an equal distribution of the hesitation index into the membership and nonmembership values.

Now, we will define the BB-entropy of an AIFS as the AIF-divergence between the AIFS and its closest 


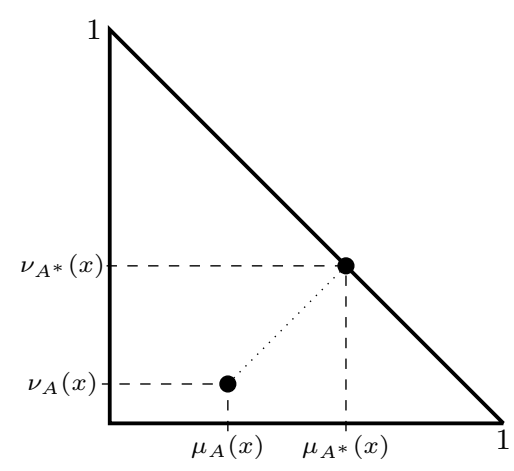

Fig. 5: Closest fuzzy set to an AIFS.

fuzzy set. Therefore, the domain of the function $h_{\mathrm{IF}}$ associated with the local AIF-divergence $D$ will be

$\mathcal{D}_{3}=\left\{\left(u, v, \frac{1+u-v}{2}, \frac{1+v-u}{2}\right) \mid u+v \leq 1\right\}$.

Theorem 4 Consider a local AIF-divergence $D$ with associated function $h_{\mathrm{IF}}$, and define the function $I$ by:

$$
\begin{aligned}
I(A) & =D\left(A, A^{*}\right) \\
& =\frac{1}{n} \sum_{x \in X} h_{\mathrm{IF}}\left(\mu_{A}(x), \nu_{A}(x), \mu_{A^{*}}(x), 1-\mu_{A^{*}}(x)\right) .
\end{aligned}
$$

Then, $I$ is a BB-entropy if and only if $h_{\mathrm{IF}}$ satisfies the following additional properties:

(AIF-loc. 1") For $\left(u, v, \frac{1+u-v}{2}, \frac{1+v-u}{2}\right) \in \mathcal{D}_{3}$, it holds that $h_{\mathrm{IF}}\left(u, v, \frac{1+u-v}{2}, \frac{1+v-u}{2}\right)=0$ if and only if $u+$ $v=1$.

(AIF-loc.7) The function $h^{*}$ defined by

$$
h^{*}(u, v)=h_{\mathrm{IF}}\left(u, v, \frac{1+u-v}{2}, \frac{1+v-u}{2}\right),
$$

for $u+v \leq 1$, is symmetric and decreasing in both $u$ and $v$.

(AIF-loc.8) $h_{\mathrm{IF}}\left(u, v, \frac{1+u-v}{2}, \frac{1+v-u}{2}\right)=1$ for $u+v \leq 1$ if and only if $u=v=0$.

Proof $\left(\mathbf{I}_{\mathbf{B B}} \mathbf{1}\right)$ : By definition, $I(A)=0$ if and only if $h_{\mathrm{IF}}\left(\mu_{A}(x), \nu_{A}(x), \mu_{A^{*}}(x), 1-\mu_{A^{*}}(x)\right)=0$ for any $x \in$ $X$, but from (AIF-loc.1") this is equivalent to $\mu_{A}(x)+$ $\nu_{A}(x)=1$ for any $x \in X$, or in other words, $A$ is a fuzzy set.

$\left(\mathbf{I}_{\mathbf{B B}} \mathbf{2}\right)$ : By definition, $I(A)=1$ if and only if

$h_{\mathrm{IF}}\left(\mu_{A}(x), \nu_{A}(x), \mu_{A^{*}}(x), 1-\mu_{A^{*}}(x)\right)=1$

for any $x \in X$, and by (AIF-loc.8) this is equivalent to $\mu_{A}(x)=\nu_{A}(x)=0$ for any $x \in X$.
$\left(\mathbf{I}_{\mathbf{B B}} \mathbf{3}\right):$ In order to prove that $I(A)=I\left(A^{c}\right)$, it is enough to realize that, by (AIF-loc.7), it holds that:

$$
\begin{aligned}
h_{\mathrm{IF}}\left(\mu_{A}(x), \nu_{A}(x), \mu_{A^{*}}(x), 1-\mu_{A^{*}}(x)\right) \\
=h_{\mathrm{IF}}\left(\nu_{A}(x), \mu_{A}(x), \mu_{A^{c *}}(x), 1-\mu_{A^{c *}}(x)\right),
\end{aligned}
$$

and that

$$
\begin{aligned}
\mu_{A^{c *}}(x) & =\mu_{A^{c}}(x)+\frac{\pi_{A^{c}}(x)}{2} \\
& =\nu_{A}(x)+\frac{\pi_{A}(x)}{2}=\nu_{A^{*}}(x) . \\
\nu_{A^{c *}}(x) & =\nu_{A^{c}}(x)+\frac{\pi_{A^{c}}(x)}{2} \\
& =\mu_{A}(x)+\frac{\pi_{A}(x)}{2}=\mu_{A^{*}}(x) .
\end{aligned}
$$

$\left(\mathbf{I}_{\mathbf{B B}} 4\right)$ : Assume that $\mu_{A}(x) \leq \mu_{B}(x)$ and $\nu_{A}(x) \leq$ $\nu_{B}(x)$. Then:

$$
\begin{aligned}
& h_{\mathrm{IF}}\left(\mu_{A}(x), \nu_{A}(x), \mu_{A^{*}}(x), 1-\mu_{A^{*}}(x)\right) \\
& =h_{\mathrm{IF}}\left(\mu_{A}(x), \nu_{A}(x), \frac{1+\mu_{A}(x)-\nu_{A}(x)}{2}, \frac{1+\nu_{A}(x)-\mu_{A}(x)}{2}\right) \\
& \geq h_{\mathrm{IF}}\left(\mu_{B}(x), \nu_{A}(x), \frac{1+\mu_{B}(x)-\nu_{A}(x)}{2}, \frac{1+\nu_{A}(x)-\mu_{B}(x)}{2}\right) \\
& \geq h_{\mathrm{IF}}\left(\mu_{B}(x), \nu_{B}(x), \frac{1+\mu_{B}(x)-\nu_{B}(x)}{2}, \frac{1+\nu_{B}(x)-\mu_{B}(x)}{2}\right) \\
& =h_{\mathrm{IF}}\left(\mu_{B}(x), \nu_{B}(x), \mu_{B^{*}}(x), 1-\mu_{B^{*}}(x)\right),
\end{aligned}
$$

where the inequalities follows from (AIF-loc.7).

On the other hand, assume that $I$ is a BB-entropy and let us prove that $h_{\mathrm{IF}}$ satisfies the additional conditions.

(AIF-loc.1"): Take $u, v$ such that $u+v \leq 1$, and define the AIFS $A$ on $\{x\}$ by $A=\langle(x, u, v)\rangle$. Then, it holds that:

$I(A)=h_{\mathrm{IF}}\left(u, v, \frac{1+u-v}{2}, \frac{1+v-u}{2}\right)$,

but from $\left(I_{\mathbf{B B}} 1\right), I(A)=1$ if and only if $u=v=0$.

(AIF-loc.7): Let us prove that $h^{*}$ is symmetric. Take $u, v$ such that $u+v \leq 1$, and define $A=\langle(x, u, v)\rangle$. From $\left(I_{\mathbf{B B}} 3\right)$, it holds that:

$$
\begin{aligned}
h^{*}(u, v) & =h_{\mathrm{IF}}\left(u, v, \frac{1+u-v}{2}, \frac{1+v-u}{2}\right) \\
& =I(A)=I\left(A^{c}\right) \\
& =h_{\mathrm{IF}}\left(v, u, \frac{1+v-u}{2}, \frac{1+u-v}{2}\right)=h^{*}(v, u) .
\end{aligned}
$$

Let us now see that $h^{*}$ is decreasing in the first component. Take $u_{1}, u_{2}, v$ such that $u_{1} \leq u_{2}$ and $u_{2}+v \leq 1$ 
and define the AIFSs $A, B$ on $\{x\}$ by $A=\left\langle\left(x, u_{1}, v\right)\right\rangle$ and $B=\left\langle\left(x, u_{2}, v\right)\right\rangle$. Then, from $\left(I_{\mathrm{BB}} 4\right)$ it holds that:

$$
\begin{aligned}
& h^{*}\left(u_{1}, v\right)=h_{\mathrm{IF}}\left(u_{1}, v, \frac{1+u_{1}-v}{2}, \frac{1+v-u_{1}}{2}\right) \\
& \quad=I(A) \geq I(B) \\
& \quad=h_{\mathrm{IF}}\left(u_{1}, v, \frac{1+u_{2}-v}{2}, \frac{1+v-u_{2}}{2}\right)=h^{*}\left(u_{2}, v\right) .
\end{aligned}
$$

With a similar reasoning we can proof that $h^{*}$ is also decreasing in the second component.

(AIF-loc.7): Take $u, v$ such that $u+v \leq 1$, and define the AIFS $A$ on $\{x\}$ by $A=\langle(x, u, v)\rangle$. Then:

$I(A)=\left(u, v, \frac{1+u-v}{2}, \frac{1+v-u}{2}\right)$,

but from $\left(I_{\mathrm{BB}} 2\right), I(A)=1$ if and only if $u=v=0$.

There is an alternative way of defining a BB-entropy using AIF-divergences. This second approach is based on the comparison of the AIFS $A$ with the fuzzy sets $A^{+}$, with membership function $\mu_{A^{+}}(x)=\mu_{A}(x)$, and $A^{-}$, with membership function $\mu_{A^{-}}(X)=\nu_{A}(x)$. We first compute the AIF-divergence between $A$ and $A^{+}$and between $A$ and $A^{-}$, and then aggregating both values. The fuzzy set, $A^{+}$and $A^{-}$, along with their associated AIFS, A, are graphically shown in Figure 6.

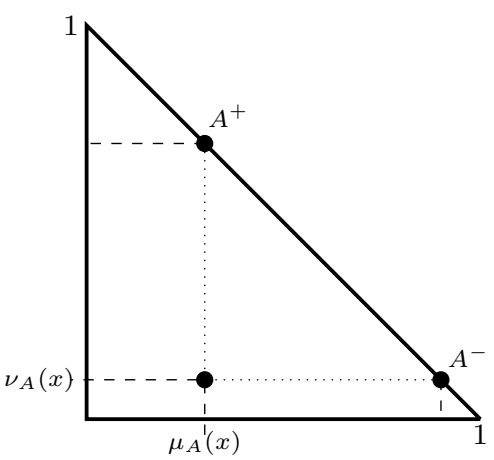

Fig. 6: Graphical representation of the definition of the sets $A^{+}$and $A^{-}$.

For the next result, the domain $\mathcal{D}_{4}$ of the function $h_{\mathrm{IF}}$ is given by:

$\mathcal{D}_{4}=\{(u, v, u, 1-u) \mid u+v \leq 1\} \cup\{(u, v, 1-v, v) \mid u+v \leq 1\}$

Proposition 4 Consider a local AIF-divergence D with associated function $h_{\mathrm{IF}}$ satisfying the following additional properties on $\mathcal{D}_{4}$ :

(AIF-loc.1"') $h_{\mathrm{IF}}\left(u_{1}, u_{2}, v_{1}, v_{2}\right)=0$ for $\left(u_{1}, u_{2}, v_{1}, v_{2}\right) \in$ $\mathcal{D}_{4}$ if and only if $u_{1}+u_{2}=1$.
(AIF-loc.5) $h_{\mathrm{IF}}\left(u_{1}, u_{2}, v_{1}, v_{2}\right)=h_{\mathrm{IF}}\left(u_{2}, u_{1}, v_{2}, v_{1}\right)$ for any $\left(u_{1}, u_{2}, v_{1}, v_{2}\right) \in \mathcal{D}_{4}$.

(AIF-loc.9) $h_{\mathrm{IF}}(u, v, u, 1-u)$ and $h_{\mathrm{IF}}(u, v, 1-v, v)$ are decreasing in both $u$ and $v$, for $u+v \leq 1$.

(AIF-loc.10) $h_{\mathrm{IF}}\left(u_{1}, u_{2}, v_{1}, v_{2}\right)=1$ for $\left(u_{1}, u_{2}, v_{1}, v_{2}\right) \in$ $\mathcal{D}_{4}$ if and only if $u_{1}=u_{2}=0$.

Consider also a function $f:[0,1] \times[0,1] \rightarrow[0,1]$ such that

(f1) $f(u, v)=0$ if and only if $u=v=0$.

(f2) $f$ is component-wise increasing.

(f5) $f(u, v)=1$ if and only if $u=v=1$.

(f6) $f(u, v)=f(v, u)$.

The function $I: \operatorname{AIFS}(X) \rightarrow[0,1]$ defined by:

$$
\begin{gathered}
I(A)=\frac{1}{n} \sum_{x \in X} f\left(h_{\mathrm{IF}}\left(\mu_{A}(x), \nu_{A}(x), \mu_{A}(x), 1-\mu_{A}(x)\right),\right. \\
\left.h_{\mathrm{IF}}\left(\mu_{A}(x), \nu_{A}(x), 1-\nu_{A}(x), \nu_{A}(x)\right)\right)
\end{gathered}
$$

is a BB-entropy.

Proof $\left(\mathbf{I}_{\mathbf{B B}} \mathbf{1}\right): I(A)=0$ if and only if

$$
\begin{aligned}
& f\left(h_{\mathrm{IF}}\left(\mu_{A}(x), \nu_{A}(x), \mu_{A}(x), 1-\mu_{A}(x)\right),\right. \\
& \left.\quad h_{\mathrm{IF}}\left(\mu_{A}(x), \nu_{A}(x), 1-\nu_{A}(x), \nu_{A}(x)\right)\right)=0
\end{aligned}
$$

for any $x \in X$. From (f1), $I(A)=0$ is equivalent to

$h_{\mathrm{IF}}\left(\mu_{A}(x), \nu_{A}(x), \mu_{A}(x), 1-\mu_{A}(x)\right)=0$

and

$h_{\mathrm{IF}}\left(\mu_{A}(x), \nu_{A}(x), 1-\nu_{A}(x), \nu_{A}(x)\right)=0$,

which by (AIF-loc.1"') is equivalent to $\mu_{A}(x)+\nu_{A}(x)=$ 1 for any $x \in X$, or equivalent, if $A$ is a fuzzy set.

$\left(\mathbf{I}_{\mathrm{BB}} \mathbf{2}\right): I(A)=1$ if and only if

$$
\begin{aligned}
& f\left(h_{\mathrm{IF}}\left(\mu_{A}(x), \nu_{A}(x), \mu_{A}(x), 1-\mu_{A}(x)\right),\right. \\
& \left.h_{\mathrm{IF}}\left(\mu_{A}(x), \nu_{A}(x), 1-\nu_{A}(x), \nu_{A}(x)\right)\right)=1,
\end{aligned}
$$

for any $x \in X$. Also, from (f5), $I(A)=1$ is equivalent to

$h_{\mathrm{IF}}\left(\mu_{A}(x), \nu_{A}(x), \mu_{A}(x), 1-\mu_{A}(x)\right)=1$

and

$h_{\mathrm{IF}}\left(\mu_{A}(x), \nu_{A}(x), 1-\nu_{A}(x), \nu_{A}(x)\right)=1$,

which by (AIF-loc.10) holds if and only if $\mu_{A}(x)=$ $\nu_{A}(x)=0$ for any $x \in X$. 
$\left(\mathbf{I}_{\mathrm{BB}} \mathbf{3}\right):$ In order to check the equality $I(A)=I\left(A^{c}\right)$, we only need to prove that

$$
\begin{aligned}
& f\left(h_{\mathrm{IF}}\left(\mu_{A}(x), \nu_{A}(x), 1-\mu_{A}(x), \nu_{A}(x)\right),\right. \\
& \left.\quad h_{\mathrm{IF}}\left(\mu_{A}(x), \nu_{A}(x), 1-\nu_{A}(x), \mu_{A}(x)\right)\right)= \\
& f\left(h _ { \mathrm { IF } } \left(\mu_{A^{c}}(x), \nu_{A^{c}}(x), \mu_{A^{c}}(x),\right.\right. \\
& \left.\left.\quad 1-\mu_{A^{c}}(x)\right), h_{\mathrm{IF}}\left(\mu_{A^{c}}(x), \nu_{A^{c}}(x), 1-\nu_{A^{c}}(x), \nu_{A^{c}}(x)\right)\right)
\end{aligned}
$$

for any $x \in X$. For this, note that from (AIF-loc.5), it holds that:

$$
\begin{aligned}
h_{\mathrm{IF}}\left(\mu_{A^{c}}(x),\right. & \left.\nu_{A^{c}}(x), \mu_{A^{c}}(x), 1-\mu_{A^{c}}(x)\right) \\
& =h_{\mathrm{IF}}\left(\nu_{A}(x), \mu_{A}(x), \nu_{A}(x), 1-\nu_{A}(x)\right) \\
& =h_{\mathrm{IF}}\left(\mu_{A}(x), \nu_{A}(x), 1-\nu_{A}(x), \nu_{A}(x)\right) .
\end{aligned}
$$

$h_{\mathrm{IF}}\left(\mu_{A^{c}}(x), \nu_{A^{c}}(x), 1-\nu_{A^{c}}(x), \nu_{A^{c}}(x)\right)$

$$
\begin{aligned}
& =h_{\mathrm{IF}}\left(\nu_{A}(x), \mu_{A}(x), 1-\mu_{A}(x), \mu_{A}(x)\right) \\
& =h_{\mathrm{IF}}\left(\mu_{A}(x), \nu_{A}(x), \nu_{A}(x), 1-\mu_{A}(x)\right) .
\end{aligned}
$$

Therefore, taking (f6) into account, it holds that:

$$
\begin{gathered}
f\left(h_{\mathrm{IF}}\left(\mu_{A}(x), \nu_{A}(x), \mu_{A}(x), 1-\mu_{A}(x)\right),\right. \\
\left.h_{\mathrm{IF}}\left(\mu_{A}(x), \nu_{A}(x), 1-\nu_{A}(x), \nu_{A}(x)\right)\right) \\
=f\left(h_{\mathrm{IF}}\left(\mu_{A^{c}}(x), \nu_{A^{c}}(x), 1-\nu_{A^{c}}(x), \nu_{A^{c}}(x)\right),\right. \\
\left.h_{\mathrm{IF}}\left(\mu_{A^{c}}(x), \nu_{A^{c}}(x), \mu_{A^{c}}(x), 1-\mu_{A^{c}}(x)\right)\right) \\
=f\left(h_{\mathrm{IF}}\left(\mu_{A^{c}}(x), \nu_{A^{c}}(x), \mu_{A^{c}}(x), 1-\mu_{A^{c}}(x)\right),\right. \\
\left.h_{\mathrm{IF}}\left(\mu_{A^{c}}(x), \nu_{A^{c}}(x), 1-\nu_{A^{c}}(x), \nu_{A^{c}}(x)\right)\right) .
\end{gathered}
$$

$\left(\mathbf{I}_{\mathbf{B B}} 4\right)$ : Take $A, B \in \operatorname{AIFS}(X)$ such that $\mu_{A} \leq \mu_{B}$ and $\nu_{A} \leq \nu_{B}$. By property (AIF-loc.9), it holds that:

$$
\begin{aligned}
& h_{\mathrm{IF}}\left(\mu_{A}(x), \nu_{A}(x), \mu_{A}(x), 1-\mu_{A}(x)\right) \\
& \geq h_{\mathrm{IF}}\left(\mu_{B}(x), \nu_{A}(x), \mu_{B}(x), 1-\mu_{B}(x)\right) \\
& \geq h_{\mathrm{IF}}\left(\mu_{B}(x), \nu_{B}(x), \mu_{B}(x), 1-\mu_{B}(x)\right) . \\
& h_{\mathrm{IF}}\left(\mu_{A}(x), \nu_{A}(x), 1-\nu_{A}(x), \nu_{A}(x)\right) \\
& \geq h_{\mathrm{IF}}\left(\mu_{B}(x), \nu_{A}(x), 1-\nu_{A}(x), n u A(x)\right) \\
& \geq h_{\mathrm{IF}}\left(\mu_{B}(x), \nu_{B}(x), 1-\nu_{B}(x), \nu_{B}(x)\right) .
\end{aligned}
$$

Therefore, using (f2) we conclude that:

$$
\begin{gathered}
f\left(h_{\mathrm{IF}}\left(\mu_{A}(x), \nu_{A}(x), \mu_{A}(x), 1-\mu_{A}(x)\right),\right. \\
\left.h_{\mathrm{IF}}\left(\mu_{A}(x), \nu_{A}(x), 1-\nu_{A}(x), \nu_{A}(x)\right)\right) \geq \\
f\left(h_{\mathrm{IF}}\left(\mu_{B}(x), \nu_{B}(x), \mu_{B}(x), 1-\mu_{B}(x)\right),\right. \\
\left.h_{\mathrm{IF}}\left(\mu_{B}(x), \nu_{B}(x), 1-\nu_{B}(x), \nu_{B}(x)\right)\right) .
\end{gathered}
$$

Then, $I(A) \geq I(B)$.

At a first glance, one may think that the converse implication in the previous proposition also holds. That is, given a function $f$ satisfying (f1), (f2), (f5) and (f6), and $I$ defined as in Equation (14), then $I$ is a BBentropy, if and only if, $h_{\mathrm{IF}}$ satisfies properties (AIFloc.1"'), (AIF-loc.5), (AIF-loc.9) and (AIF-loc.10). However, as next example shows, the equivalence cannot be guaranteed.

Example 5 Consider the function $D$ given by:

$D(A, B)=\frac{1}{n} \sum_{x \in X}\left|\mu_{A}(x)-\mu_{B}(x)\right|+\left|\nu_{A}(x)-\nu_{B}(x)\right|^{2}$.

This function is a local AIF-divergence whose associated function $h_{\mathrm{IF}}$ is given by:

$h_{\mathrm{IF}}\left(u_{1}, u_{2}, v_{1}, v_{2}\right)=\left|u_{1}-v_{1}\right|+\left|u_{2}-v_{2}\right|^{2}$.

Applying Equation (14) to this function $h_{\mathrm{IF}}, I(A)$ is given by:

$$
\begin{aligned}
& I(A) \\
& =\frac{1}{n} \sum_{x \in X} f\left(\left|\mu_{A}(x)-\mu_{A}(x)\right|+\left|1-\mu_{A}(x)-\nu_{A}(x)\right|^{2},\right. \\
& =\frac{1}{n} \sum_{x \in X} f\left(\left|1-\mu_{A}(x)-1+\nu_{A}(x)\right|+\left|\nu_{A}(x)-\nu_{A}(x)\right|^{2}\right) \\
& =\frac{1}{n} \sum_{x \in X} f\left(\pi_{A}(x)^{2},\left|1-\mu_{A}(x)-\nu_{A}(x)\right|\right) .
\end{aligned}
$$

Consider the function $f:[0,1] \times[0,1] \rightarrow[0,1]$ given by $f(x, y)=\frac{x+y}{2}$, which satisfies (f1), (f2), (f5) and (f6). Substituting in Equation (15), we obtain the following:

$$
\begin{aligned}
I(A) & =\frac{1}{n} \sum_{x \in X} \frac{\pi_{A}(x)+\pi_{A}(x)^{2}}{2} \\
& =\frac{1}{2 n} \sum_{x \in X} \pi_{A}(x)\left(1+\pi_{A}(x)\right) .
\end{aligned}
$$

This function is a BB-entropy:

$\left(\mathbf{I}_{\mathrm{BB}} \mathbf{1}\right): I(A)=0$ if and only if $\pi_{A}(x)\left(1+\pi_{A}(x)\right)=$ 0 for any $x \in X$, but this is equivalent to $\pi(A)=0$ for any $x \in X$, so $A$ is a fuzzy set.

$\left(\mathbf{I}_{\mathrm{BB}} \mathbf{2}\right): I(A)=1$ if and only if $\frac{1}{2} \pi_{A}(x)\left(1+\pi_{A}(x)\right)=$ 1 for any $x \in X$, but this is equivalent to $\pi_{A}(x)=1$ for any $x \in X$, so $\mu_{A}=\nu_{A}=0$.

$\left(\mathbf{I}_{\mathrm{BB}} \mathbf{3}\right)$ : Trivially, $I(A)=I\left(A^{c}\right)$ holds.

$\left(\mathbf{I}_{\mathrm{BB}} 4\right)$ : Take $\mu_{A} \leq \mu_{B}$ and $\nu_{A} \leq \nu_{B}$. This implies that $\nu_{A} \geq \nu_{B}$, and therefore $\pi_{A}\left(1+\pi_{A}\right) \geq \pi_{B}\left(1+\pi_{B}\right)$, which implies that $I(A) \geq I(B)$.

We conclude that $I$ is a BB-entropy. However, the function $h_{\mathrm{IF}}$ does not satisfy property (AIF-loc.5): take $(0.6,0.1,0.6,0.4),(0.1,0.6,0.4,0.6) \in \mathcal{D}_{4}$. It holds that:

$h_{\mathrm{IF}}(0.6,0.1,0.6,0.4)=|0.6-0.6|+|0.1-0.4|^{2}=0.09$. $h_{\mathrm{IF}}(0.1,0.6,0.4,0.6)=|0.1-0.4|+|0.6-0.6|^{2}=0.3$.

Since both values do not coincide, $h_{\mathrm{IF}}$ does not satisfy property (AIF-loc.5). 
We conclude that the sufficient conditions given in Proposition 4 are not necessary.

\subsection{Examples of BB-entropies based on} AIF-divergences

We consider again the four local AIF-divergences defined in Subsection 3.3, the Hamming and Hausdorff distances $l_{A I F}, d_{H}$ and the AIF-divergences defined by Hong and Kim $D_{C}, D_{L}$. In order to make them to satisfy the normalization properties mentioned in Subsection 2.3 , in this section we consider also $d_{H}^{*}$, which is defined by $d_{H}^{*}=2 d_{H}$. We first apply Theorem 4 to these four local AIF-divergences.

Proposition 5 Consider the AIF-divergences $l_{A I F}, d_{H}^{*}$, $D_{C}$ and $D_{L}$. They satisfy the conditions of Theorem 4, so they induce a BB-entropy. Also, the BB-entropies they induce coincide and it is given by:

$I(A)=\frac{1}{n} \sum_{x \in X} \pi_{A}(x)$.

Proof First of all, let us see that $h_{1}, h_{2}$ and $h_{3}$ coincide in the domain $\mathcal{D}_{3}$ :

$$
\begin{array}{r}
h_{1}\left(u, v, \frac{1+u-v}{2}, \frac{1+v-u}{2}\right) \\
=\frac{1}{2}\left(\left|\frac{1-u-v}{2}\right|+\left|\frac{1-u-v}{2}\right|+|1-u-v|\right) \\
=1-u-v . \\
h_{2}\left(u, v, \frac{1+u-v}{2}, \frac{1+v-u}{2}\right) \\
=2 \max \left\{\frac{1-u-v}{2}, \frac{1-u-v}{2}\right\}=1-u-v . \\
h_{3}\left(u, v, \frac{1+u-v}{2}, \frac{1+v-u}{2}\right) \\
=\left|\frac{1-u-v}{2}\right|+\left|\frac{1-u-v}{2}\right|=1-u-v . \\
h_{4}\left(u, v, \frac{1+u-v}{2}, \frac{1-u+v}{2}\right) \\
=\frac{1}{2}\left(\left|u-\frac{1+u-v}{2}-v+\frac{1-u+v}{2}\right|\right. \\
\left.+\left|\frac{1-u-v}{2}\right|+\left|\frac{1-u-v}{2}\right|\right)=1-u-v .
\end{array}
$$

Thus, we see that all $h_{l}, h_{d *}, h_{C}, h_{L}$ coincide in $\mathcal{D}_{3}$. Now, let us see that they satisfy the conditions on Theorem 4.

$\left(\mathbf{A I F}-\right.$ loc. $\left.\mathbf{1}^{\prime \prime}\right):$ It holds that:

$h_{l}\left(u, v, \frac{1+u-v}{2}, \frac{1-u+v}{2}\right)=1-u-v=0$ if and only if $u+v=1$.

$\left(\right.$ AIF - loc.7) $: h_{l}^{*}(u, v)=1-u-v$, so obviously $h_{l}^{*}$ is symmetric and decreasing in both $u, v$.

(AIF - loc.8) : It holds that:

$h_{l}\left(u, v, \frac{1+u-v}{2}, \frac{1-u+v}{2}\right)=1-u-v=1$

if and only if $u=v=0$.

Therefore, $h_{l}, h_{d *}, h_{C}$, all satisfy the conditions of Theorem 4, so each of Hamming and Hausdorff distances and $D_{C}, D_{L}$, induces a BB-entropy measure. Furthermore, since $h_{l}, h_{d *}, h_{C}, h_{L}$ coincide in $\mathcal{D}_{3}$, all of them induce the same BB-entropy. Using Equation (13), we obtain the following formula:

$$
\begin{aligned}
I(A) & =\frac{1}{n} \sum_{x \in X} h\left(\mu_{A}(x), \nu_{A}(x), \mu_{A^{*}}(x), 1-\mu_{A^{*}}(x)\right) \\
& =\frac{1}{n} \sum_{x \in X} \pi_{A}(x) .
\end{aligned}
$$

The BB-entropy obtained in the previous proposition has already been proposed by [?] and used in other papers like [?].

Let us now apply the procedure described in Proposition 5 to the four local AIF-divergences. As next result shows, the four measures induce again the same BB-entropy.

Proposition 6 Consider the four local AIF-divergences $l_{A I F}, d_{H}, D_{C}, D_{L}$ and a function $f:[0,1] \times[0,1] \rightarrow$ $[0,1]$ satisfying properties (f1), (f5) and (f6). Then, these four local AIF-divergences satisfy the conditions of Proposition 5, so then each of them induces a BB-entropy. Indeed, they induce the same BB-entropy, given by:

$$
I(A)=\frac{1}{n} \sum_{x \in X} f\left(\pi_{A}(x), \pi_{A}(x)\right) .
$$

Proof First of all, let us see that $h_{1}, h_{2}, h_{3}, h_{4}$ coincide in $\mathcal{D}_{4}$ and that they take the value $1-u-v$.

$$
\begin{aligned}
& h_{l}(u, v, u, 1-u) \\
& \quad=\frac{1}{2}(|u-u|+|1-u-v|+|1-u-v|) \\
& \quad=1-u-v . \\
& h_{d *}(u, v, u, 1-u)=\max \{|u-u|,|1-u-v|\} \\
& \quad=1-u-v . \\
& h_{C}(u, v, u, 1-u)=|u-u|+|1-u-v|=1-u-v . \\
& h_{L}(u, v, u, 1-u) \\
& \quad=\frac{1}{2}(|u-u-(1-u)+v|+|u-u|+|1-u-v|) \\
& \quad=1-u-v .
\end{aligned}
$$


Similarly, it can be seen that:

$$
\begin{aligned}
& h_{l}(u, v, 1-v, v)=h_{d *}(u, v, 1-v, v) \\
& \quad=h_{C}(u, v, 1-v, v)=h_{L}(u, v, 1-v, v)=1-u-v .
\end{aligned}
$$

Next we shows that they satisfy the conditions on Proposition 5:

$\left(\mathbf{A I F}-\operatorname{loc} . \mathbf{1}^{\prime \prime \prime}\right): h_{l}(u, v, u, 1-u)=h_{l}(u, v, 1-v, u)=$ $1-u-v=0$ if and only if $u+v=1$.

$\left(\right.$ AIF - loc.5) $: h_{l}$ is trivially symmetric in the domain $\mathcal{D}_{4}$ since both $h_{l}(u, v, u, 1-u)$ and $h_{l}(u, v, 1-v, v)$ coincide and take the value $1-u-v$.

(AIF - loc.9) : Again, since

$h_{l}(u, v, u, 1-u)=h_{l}(u, v, 1-v, v)=1-u-v$,

so it is always decreasing in both $u$ and $v$.

$\left(\mathbf{A I F}-\right.$ loc.10) $: h_{l}(u, v, u, 1-u)=h_{l}(u, v, 1-v, v)=$ $1-u-v=1$ if and only if $u=v=0$.

We can see that $h_{l}$, and in a similar manner also $h_{d *}, h_{C}, h_{L}$, satisfy the properties of Proposition 5 , and therefore they define a BB-entropy which is given by:

$$
\begin{aligned}
I(A) & =\frac{1}{n} \sum_{x \in X} f\left(h_{l}\left(\mu_{A}(x), \nu_{A}(x), \mu_{A}(x), 1-\mu_{A}(x)\right),\right. \\
& \left.=\frac{1}{n} \sum_{x \in X} f\left(\mu_{A}(x), \nu_{A}(x), 1-\nu_{A}(x), \nu_{A}(x)\right)\right)
\end{aligned}
$$

If we consider the particular functions:

$f_{1}(u, v)=\sqrt{u \cdot v}, \quad f_{2}(u, v)=1-(1-\sqrt{u \cdot v})^{k}$, $f_{3}(u, v)=\frac{1}{e} \sqrt{u \cdot v} \cdot e^{\sqrt{u \cdot v}}$

where $k$ is an integer, we obtain the following BBentropies:

$$
\begin{aligned}
& I_{1}(A)=\frac{1}{n} \sum_{x \in X} \pi_{A}(x) . \\
& I_{2}(A)=\frac{1}{n} \sum_{x \in X}\left(1-\left(1-\pi_{A}(x)\right)^{k}\right) . \\
& I_{3}(A)=\frac{1}{e n} \sum_{x \in X} \pi_{A}(x) e^{\pi_{A}(x)} .
\end{aligned}
$$

The former has already been obtained in Proposition 6, while the second and the third were already presented as examples of BB-entropies in [?].

\section{Knowledge Measures}

In a recent paper [?], the notion of knowledge measure was introduced by Guo. The aim of this measure is to quantify the amount of knowledge conveyed by an AIFS.

Definition 7 ([?]) A mapping $K: \operatorname{AIFS}(X) \rightarrow[0,1]$ is called a knowledge measure if $K$ has the following properties:

(KP1) $K(A)=1$ if and only if $A$ is crisp.

(KP2) $K(A)=0$ if and only if $\pi_{A}=1$.

(KP3) $K\left(A^{c}\right)=K(A)$.

(KP4) $K(A) \geq K(B)$ if $\mu_{A}(x) \leq \mu_{B}(x)<\nu_{B}(x) \leq$ $\nu_{A}(x)$ or $\nu_{A}(x) \leq \nu_{B}(x) \leq \mu_{B}(x) \leq \mu_{A}(x)$ for any $x \in X$.

We can simply notice that a knowledge measure is nothing but the complementary of an SK-entropy: $K$ is a knowledge measure if and only if $E=1-K$ is a SKentropy. In this way, we can simply adapt our results from Section 3 to build knowledge measures using local AIF-divergences. For instance, we can easily write Theorem 3 in terms of knowledge measures:

Corollary 1 Consider a local AIF-divergence $D$ with associated function $h_{\mathrm{IF}}$, and define the function $K$ by:

$$
\begin{aligned}
K(A) & =1-D\left(A, C_{A}\right) \\
& =1-\frac{1}{n} \sum_{x \in X} h_{\mathrm{IF}}\left(\mu_{A}(x), \nu_{A}(x), \mu_{C_{A}}(x), \nu_{C_{A}}(x)\right)
\end{aligned}
$$

for any $A \in \operatorname{AIFS}(X)$. Then, $K$ is a knowledge measure if and only if $h_{\mathrm{IF}}$ satisfies the conditions (AIF-loc.1'), (AIF-loc.5) and (AIF-loc.6).

The proof is analogous to that of Theorem 3 and therefore, omitted. Also, we can adapt the other results from this section, as well as, the examples given in Subsection 3.3, which give rise to the following knowledge measures:

$$
\begin{aligned}
K_{1}(A)=\frac{1}{n} \sum_{x \in X} \max \left\{\mu_{A}(x), \nu_{A}(x)\right\} . \\
K_{2}(A)=1-\frac{1}{n} \sum_{x \in X}\left(1-\mid \mu_{A}(x)\right. \\
\left.\quad-\nu_{A}(x) \mid\right)\left(\frac{2-\mu_{A}(x)-\nu_{A}(x)}{2}\right) . \\
K_{3}(A)=1-\frac{1}{n} \sum_{x \in X}\left(\left(1-\mu_{A}(x)\right)\left(1-\nu_{A}(x)\right)\right. \\
\left.\quad+\min \left\{\mu_{A}(x), \nu_{A}(x)\right\}\right) .
\end{aligned}
$$

$K_{1}$ is a knowledge measure that can be built using the Hamming and Hausdorff distances. $K_{2}$ is a knowledge 
measure already mentioned in [?,?], while $K_{3}$ is another knowledge measure that can built using the Hamming distance for fuzzy sets as we did in Example 3.

\section{Application to multi-attribute group decision making}

In this section we present an application of our results in a multi-attribute decision making (MADM, for short) problem. In detail, we continue with the approach given in [?, Section 6], where knowledge measures were used to obtain the weights of the experts.

First of all, let us introduce the main notations. In MADM problems, there are a number of alternatives $X=\left\{x_{1}, \ldots, x_{n}\right\}$ and $A=\left\{a_{1}, \ldots, a_{m}\right\}$ are the attributes with a weight vector $w=\left(w_{1}, \ldots, w_{m}\right)$. The alternatives are evaluated by some experts $e_{1}, \ldots, e_{l}$. Their evaluations of the alternatives are given as IFSs in a matrix form: $S^{(k)}$ is a $n \times m$ matrix such that $s_{i, j}^{(k)}=\left\langle x_{i}, \mu_{i, j}^{(k)}, \nu_{i, j}^{(k)}\right\rangle$ denotes the IFS that represents the evaluation of the expert $k$ of the alternative $x_{i}$ on the attribute $a_{j}$.

In [?], Nguyen used knowledge measures to determine weight vector for the experts, following these steps:

- Step 1: For each expert $e_{k}$, we compute the individual overall evaluation values of alternative $x_{i}$ by using the following intuitionistic fuzzy weighted averaging operator [?]:

$$
z_{i}^{(k)}=\left\langle x_{i}, 1-\prod_{j=1}^{5}\left(1-\mu_{i, j}^{(k)}\right)^{w_{j}}, \prod_{j=1}^{m}\left(\nu_{i, j}^{(k)}\right)^{w_{j}}\right\rangle,
$$

- Step 2: For each expert $e_{k}$, we compute the knowledge of its overall evaluation $z_{i}$, denoted by $K\left(z^{(k)}\right)$.

- Step 3: We define the weights of the experts by normalizing the values $K\left(z^{(i)}\right)$ :

$$
\lambda_{k}=\frac{K\left(z^{(k)}\right)}{\sum_{i=1}^{l} K\left(z^{(i)}\right)} .
$$

From our comments in Section 5 we deduce that his approach is made in terms of SK-entropies, which means that it determines the weights of experts by measuring the lack of information of any expert about whether the alternative is adequate or not. Then, the smaller the lack of information, the greater the weight. However, as we have explained before, we could also use BB-entropies instead of SK-entropies to measure the indecision of the experts.

Hence, we propose to modify the previous procedure as follows:
- Step 2*: Let us fix a local AIF-divergence and the SK- and BB-entropies it defines, denoted by $E_{S K}$ and $E_{B B}$. For each expert $e_{k}$, we compute the SKand BB-entropy of its overall evaluation: $E_{S K}\left(z^{(k)}\right)$ and $E_{B B}\left(z^{(k)}\right)$.

- Step $3^{*}$ : We define the weight of each expert by normalizing the entropies:

$$
\begin{aligned}
\alpha_{k} & =\frac{1-E_{S K}\left(z^{(k)}\right)}{\sum_{i=1}^{l}\left(1-1-E_{S K}\left(z^{(k)}\right)\right)}, \\
\beta_{k} & =\frac{1-E_{B B}\left(z^{(k)}\right)}{\sum_{i=1}^{l}\left(1-1-E_{B B}\left(z^{(k)}\right)\right)} .
\end{aligned}
$$

Once that we have obtained these values, we can proceed as follows:

1. The weights $\alpha_{k}$ are computed by measuring the lack of information of any expert about whether the alternative is adequate or not. Then, the weight decreases as the lack of information decreases.

2 . The weights $\beta_{k}$ are computed by measuring the determination of the experts, in the sense that the smaller indeterminacy of the expert, the closer is $s_{i, j}^{(k)}$ to be a crisp set, so the greater the weight.

Our framework includes the approach of Nguyen [?] as a particular case, just following the first interpretation and taking into account our comments in Section 5 .

We next apply this approach in the following example, which first appeared in [?, Example 4].

Example 6 Consider the MADM problem that consists in choosing an air-conditioning system between three alternatives $x_{1}, x_{2}, x_{3}$. In order to take the decision, five attributes are analyzed: good quality $\left(a_{1}\right)$, easiness to operate $\left(a_{2}\right)$, being economical $\left(a_{3}\right)$, good service $\left(a_{4}\right)$ and price $\left(a_{5}\right)$, where their weight vector is $w=$ $(0.2,0.299,0.106,0.156,0.239)$. Three experts $e_{1}, e_{2}, e_{3}$ evaluate the alternatives and they give the following IFSs:

$S^{(1)}=\left(\begin{array}{ccccc}\langle 0.8,0.1\rangle & \langle 0.7,0.1\rangle & \langle 0.7,0.2\rangle & \langle 0.9,0\rangle & \langle 0.5,0.4\rangle \\ \langle 0.7,0.1\rangle & \langle 0.8,0.2\rangle & \langle 0.6,0.4\rangle & \langle 0.7,0.1\rangle & \langle 0.4,0.6\rangle \\ \langle 0.8,0.2\rangle & \langle 0.9,0.1\rangle & \langle 0.7,0\rangle & \langle 0.7,0.2\rangle & \langle 0.5,0.5\rangle\end{array}\right)$

$S^{(2)}=\left(\begin{array}{ccccc}\langle 0.9,0.1\rangle & \langle 0.8,0.1\rangle & \langle 0.7,0\rangle & \langle 0.9,0.1\rangle & \langle 0.7,0.3\rangle \\ \langle 0.7,0.2\rangle & \langle 0.8,0.1\rangle & \langle 0.9,0.1\rangle & \langle 0.7,0.3\rangle & \langle 0.7,0.2\rangle \\ \langle 0.7,0.1\rangle & \langle 0.9,0\rangle & \langle 0.8,0\rangle & \langle 0.8,0.2\rangle & \langle 0.3,0.6\rangle\end{array}\right)$

$S^{(3)}=\left(\begin{array}{ccccc}\langle 0.8,0\rangle & \langle 0.7,0.1\rangle & \langle 0.9,0\rangle & \langle 0.8,0.1\rangle & \langle 0.6,0.4\rangle \\ \langle 0.8,0.2\rangle & \langle 0.7,0.3\rangle & \langle 0.8,0.1\rangle & \langle 0.9,0.1\rangle & \langle 0.3,0.6\rangle \\ \langle 0.9,0.1\rangle & \langle 0.8,0\rangle & \langle 0.8,0.1\rangle & \langle 0.9,0\rangle & \langle 0.4,0.5\rangle\end{array}\right)$

Using Step 2, the individual overall evaluation of the experts are given by:

$$
\begin{aligned}
& z_{1}=\left\langle\left(x_{1}, 0.737,0\right),\left(x_{2}, 0.677,0.219\right),\left(x_{3}, 0.775,0\right)\right\rangle . \\
& z_{2}=\left\langle\left(x_{1}, 0.82,0\right),\left(x_{2}, 0.701,0.217\right),\left(x_{3}, 0.7625,0\right)\right\rangle . \\
& z_{3}=\left\langle\left(x_{1}, 0.752,0\right),\left(x_{2}, 0.727,0.245\right),\left(x_{3}, 0.797,0\right)\right\rangle .
\end{aligned}
$$


Now, consider the Hamming distance $l_{A I F S}$, and the SK- and BB-entropies they induce, denoted by $E_{S K}$ and $E_{B B}$, which were computed in Eqs. (11) and (16). Then, following Step $2^{*}$ we obtain the following values:

\begin{tabular}{c|ccc} 
& $z_{1}$ & $z_{2}$ & $z_{3}$ \\
\hline$E_{S K}\left(z_{i}\right)$ & 0.271 & 0.239 & 0.241 \\
$E_{B B}\left(z_{i}\right)$ & 0.204 & 0.167 & 0.160
\end{tabular}

Thus, following Step 3*, we obtain the final weight vectors:

$\alpha=(0.324,0.339,0.337), \quad \beta=(0.322,0.338,0.340)$.

Let us compare the two obtained weight vectors. On the one hand, the weight vector $\alpha$ is given in terms of the amount of knowledge of the experts. This means that expert $e_{2}$ has a slightly greater weight than $e_{3}$, and the least informative expert is $e_{1}$. On the other hand, the weight vector $\beta$ is given in terms of the determination of each expert. With respect to this second approach, $e_{3}$ is the most determined expert, so she has a slightly greater weight than $e_{2}$ and $e_{1}$.

Finally, if we want to take into account the amount of information of the experts, we shall use the weights $\alpha_{k}$, while if we want to take into account the determination of the experts, we shall use the weights $\beta_{k}$. If we want to take into account both points of view, we could aggregate both weights by means of any appropriate combination. In any case, we have to notice that both weights come from the same divergence measure, so they have a common starting point and they are related in all the cases.

As soon as we determine the weights, we can use the usual procedures from MADM to determine which is the most adequate alternative.

\section{Conclusions}

In the framework of AIFSs, two different ways of defining entropies can be found in the literature. On the one hand, Szmidt and Kacprzyk define entropy as a measure of how far an AIFS is from a fuzzy set; on the other hand, Burrillo and Bustince define entropy as a measure of how far an AIFS is from its closest fuzzy set.

In this paper, we have used local AIF-divergence measures, which are functions that measure how different two AIFSs are, to define both types of entropies. In the framework of Szmidt and Kacprzyk, we have defined the closets crisp set to an AIFS and then we have defined the SK-entropy as the AIF-divergence between an AIFS and its closest crisp set. In the framework of
Bustince and Burrillo, we have defined its closest fuzzy set, and then we have defined the BB-entropy as the AIF-divergence between the AIFS and its closest fuzzy set.

In both approaches, we have studied the properties that must be imposed in the AIF-divergence to guarantee that they define either a SK-entropy or a BBentropy. We have also seen that the usual examples of SK- and BB-entropies can be obtained using local AIF-divergences. Finally, we have also seen that SKentropies and knowledge measures are equivalent, so we can also apply our results to define knowledge measures using local AIF-divergences.

As a future research, we aim to apply entropies and knowledge measures defined from AIF-divergence measures in image processing, as was done in [?,?], or to pattern recognition, as was done in $[?, ?]$.

Acknowledgements A shorter version of this paper was published at EUSFLAT Conference [?]. The current version has been updated with additional results, comments and proofs. We acknowledge the financial support by project TIN201459543-P.

\section{Compliance with ethical standards}

Conflict of interest The authors declare that they have no conflict of interest.

Ethical approval This article does not contain any studies with human participants or animals performed by any of the authors.

\section{References}

1. Atanassov, K.: Intuitionistic fuzzy sets. Fuzzy Sets and Systems 20, 87-96 (1986)

2. Bhandari, D., Pal, N.: Some new information measures for fuzzy sets. Information Sciences 67(3), 209-228 (1993)

3. Bhandari, D., Pal, N., Majumder, D.: Fuzzy divergence, probability measure of fuzzy events and image thresholding. Pattern Recognition Letters 13(12), 857-867 (1992)

4. Burrillo, P., Bustince, H.: Entropy on intuitionistc fuzzy sets and on interval-valued fuzzy sets. Fuzzy Sets and Systems 78, 305-316 (1996)

5. De Luca, A., Termini, S.: A definition of nonprobabilistic entropy in the setting of fuzzy theory. Journal of General Systems 5, 301-312 (1972)

6. Deng, G., Jiang, Y., Fu, J.: Monotonic similarity measures between intuitionistic fuzzy sets and their relationship with entropy and inclusion measure. Information Science 316, 348-369 (2015)

7. Dubois, D., Prade, H. (eds.): Fundamentals of Fuzzy Sets. Spinger (2000)

8. Farnoosh, R., Rahimi, M., Kumar, P.: Removing noise in a digital image using a new entropy method based on intuitionistic fuzzy sets. In: 2016 IEEE International Conference on Fuzzy Systems (FUZZ-IEEE) (2016)

9. Grzegorzewski, P.: Distances between intuitionistic fuzzy sets and/or interval-valued fuzzy sets based on the Hausdorff metric. Fuzzy Sets and Systems 148, 319-328 (2004) 
10. Guo, K.: Knowledge measure for Atanassov's intuitionistic fuzzy sets. IEEE Transactions on Fuzzy Systems 24(5), 1072-1078 (2016)

11. Guo, K., Song, Q.: On the entropy for Atanassov's intuitionistic fuzzy sets: An interpretation from the perspective of amount of knowledge. Applied Soft Computing 24, 328-340 (2014)

12. Hong, D., Kim, C.: A note on similarity measures between vague sets and between elements. Information Sciences 115, 83-96 (1999)

13. Hung, W., Yang, M.: Similarity measures of intuitionistic fuzzy sets based on Hausdorff distances. Pattern Recognition Letters 25, 1603-1611 (2004)

14. Joshi, R., Kumar, S.: Parametric ( $\mathrm{r}, \mathrm{s})$-norm entropy on intuitionistic fuzzy sets with a new approach in multi attribute decision making. Fuzzy Information and Engineering 9(2), 181-203 (2017)

15. Kacprzyk, J., Pedrycz, W. (eds.): Springer Handbook of Computational Intelligence. Springer (2015)

16. Klement, E., Mesiar, R., Pap, E.: Triangular norms. Kluwer Academic Publishers (2000)

17. Kosko, B.: Fuzzy entropy and conditining. Information Sciences 40, 166-177 (1986)

18. Liang, Z., Shi, P.: Similarity measures on intuitionistic fuzzy sets. Pattern Recognition Letters 24, 2687-2693 (2003)

19. Liu, X.: Entropy, distance measure and similarity measure of fuzzy sets and their relation. Fuzzy Sets and Systems 53, 305-318 (1992)

20. Melo-Pinto, P., Couto, P., Bustince, H., Barrenechea, E., Pagola, M., Fernandez, J.: Image segmentation using Atanassov's intuitionistic fuzzy sets. Expert Systems with Applications 40(1), 15-26 (2013)

21. Meng, F., Chen, X.: Entropy and similarity measure of atanassov's intuitionistic fuzzy sets and their application to pattern recognition based on fuzzy measures. Pattern Analysis and Applications 19(1), 1120 (2016). DOI 10.1007/s10044-014-0378-6. URL https://doi.org/10.1007/s10044-014-0378-6

22. Montes, I., Janis, V., Montes, S.: An axiomatic definition of divergence for intuitionistic fuzzy sets. In: Proceedings of EUSFLAT Conference, vol. 1, pp. 547-553 (2011)

23. Montes, I., Janis, V., Montes, S.: Local IF-divergences. Communications in Computer and Information Science 298 CCIS(PART 2), 491-500 (2012)

24. Montes, I., Janis, V., Pal, N., Montes, S.: Local Divergences for Atanassov Intuitionistc Fuzzy Sets. IEEE Transactions on Fuzzy Systems 24(2), 360-373 (2016)

25. Montes, I., Montes, S., Pal, N.: On the use of divergences for defining entropies for Atanassov intuitionistic fuzzy sets. Advances in Intelligent Systems and Computing 642, 554-565 (2018)

26. Montes, I., Pal, N., Janis, V., Montes, S.: Divergence Measures for Intuitionistc Fuzzy Sets. IEEE Transactions on Fuzzy Systems 23(2), 444-456 (2015)

27. Montes, S., Couso, I., Bertoluzza, C.: Some classes of fuzziness measures from local divergences. Belgian Journal of Operations Research, Statistics and Computer Science 38(2-3), 37-49 (1998)

28. Montes, S., Couso, I., Gil, P., Bertoluzza, C.: Divergence measure between fuzzy sets. International Journal of Approximate Reasoning 30(2), 91-105 (2002)

29. Nguyen, H.: A new knowledge-based measure for intuitionistic fuzzy sets and its application in multiple attribute group decision making. Expert Systems with Applications 42, 8766-8774 (2015)
30. Pal, N., Bejdek, J.: Measuring fuzzy uncertainty. IEEE Transactions on Fuzzy Systems 2(2), 107-118 (1994)

31. Pal, N., Bustince, H., Pagola, M., Mukherjee, U., Goswami, D., Beliakov, G.: Uncertainty with Atanassov intuitionistc fuzzy sets: Fuzziness and lack of knowledge. Information Sciences 228, 61-74 (2013)

32. Szmidt, E., Kacprzyk, J.: Distances between intuitionistc fuzzy sets. Fuzzy Sets and Systems 114, 505-518 (2000)

33. Szmidt, E., Kacprzyk, J.: Entropy for intuitionistic fuzzy sets. Fuzzy Sets and Systems 118, 467-477 (2001)

34. Szmidt, E., Kacprzyk, J.: An application of intuitionistic fuzzy set similarity measures to a multi-criteria decision making problem. In: Artificial Intelligence and Soft Compyting ICAISC. Lecture Notes in Computer Science, vol. 4029, pp. 314-323. Springer (2006)

35. Szmidt, E., Kacprzyk, J., Bujnowski, P.: How to measure the amount of knowledge conveyed by Atanassov's intuitionistic fuzzy sets. Information Sciences 257, 276-285 (2014)

36. Torra, V.: Hesitant fuzzy sets. International Journal of Intelligent Systems 25, 529-539 (2010)

37. Trillas, E., Riera, T.: Entropies in finite fuzzy sets. Information Sciences 15, 159-168 (1978)

38. $\mathrm{Xu}, \mathrm{Z}$.: Some similarity measures of intuitionistic fuzzy sets and their application to multiple attribute decision making. Fuzzy Optimization and Decision Making 6, 109-121 (2007)

39. Xu, Z., Cai, X.: Nonlinear optimization models for multiple attribute group decision making with intuitionistic fuzzy information. International Journal of Intelligent Systems 25(6), 489-513 (2010)

40. Yager, R.: On measure of fuzziness and fuzzy complements. International Journal of General Systems 8, 169$180(1982)$

41. Zadeh, L.: Fuzzy sets. Information and Control 8, 338353 (1965)

42. Zadeh, L.: The concept of a linguistic variable and its application to approximate reasoning. Information Sciences 8, 199-249 (1975)

43. Zimmermann, H.J.: Fuzzy Set Theory and Its Applications. Springer (2001) 